\title{
Adaptive and Off-Line Techniques for Non-Linear Multiscale Analysis
}

\author{
Stefano Zaghi ${ }^{\mathrm{a}}$, Xavier Martinez ${ }^{\mathrm{a}, \mathrm{c}, *}$, Riccardo Rossi $^{\mathrm{a}, \mathrm{b}}$, Massimo Petracca $^{\mathrm{d}}$ \\ ${ }^{a}$ CIMNE - Centre Internacional de Metodes Numerics en Enginyeria, Universitat Politècnica de \\ Catalunya (UPC-BarcelonaTech), Barcelona 08034, Spain \\ ${ }^{b}$ Department of Civil and Environmental Engineering, Universitat Politècnica de Catalunya \\ (UPC-BarcelonaTech), Barcelona 08034, Spain \\ ${ }^{c}$ Department of Nautical Science and Engineering, Universitat Politècnica de Catalunya \\ (UPC-BarcelonaTech), Barcelona 08003, Spain \\ dDepartment of Engineering, University "G.d'Annunzio" of Chieti and Pescara, Pescara 65127, Italy
}

\begin{abstract}
This paper presents two procedures, based on the numerical multiscale theory, developed to predict the mechanical non-linear response of composite materials under monotonically increasing loads. Such procedures are designed with the objective of reducing the computational cost required in these types of analysis. Starting from virtual tests of the microscale, the solution of the macroscale structure via Classical First-Order Multiscale Method will be replaced by an interpolation of a discrete number of homogenized surfaces previously calculated. These surfaces describe the stress evolution of the microscale at fixed levels of an equivalent damage parameter $\left(d_{e q}\right)$. The information required for these surfaces to conduct the analysis is stored in a Data Base using a json format. Of the two methods developed, the first one uses the pre-computed homogenized surface just to obtain the material non-linear threshold, and generates a Representative Volume Element (RVE) once the material point goes into the nonlinear range; the second method is completely off-line and is capable of describing the material linear and non-linear behavior just by using the discrete homogenized surfaces stored in the Data Base. After describing the two procedures developed, this manuscript provides two examples to validate the capabilities of the proposed methods.
\end{abstract}

Keywords: Multiscale, Multiphysics, Optimization

\section{Introduction}

A composite material is defined as a complex structure characterized by two or more components with different mechanical, thermal and/or chemical properties. The combinations of multiple constituents leads to a new material that usually improves significantly

\footnotetext{
*corresponding author

Email addresses: zstefano@cimne.upc.edu (Stefano Zaghi), xmartinez@cimne.upc.edu (Xavier Martinez), rrossi@cimne.upc.edu (Riccardo Rossi), mpetracca@cimne.upc.edu (Massimo Petracca) 
the properties of the base materials. Examples of composites are the fiber reinforced polymers (FRP) or even the reinforced concrete used in civil engineering applications. From a numerical point of view the simulation of these non-homogeneous materials has always represented a challenge because of the interaction between the different constituent materials, associated to the usually complex composite internal micro-structure. Modelling each single component of the composite, also called Direct Numerical Simulation (DNS), provides the highest level of accuracy but, at the same time, the simulation of largescale structures implies a prohibitively expensive computational cost. For this reason, to increase the efficiency of the simulations, the non-homogeneous composite materials are often treated as homogeneous continua characterized by more complex constitutive models. The classical mixing theory [1] is one of the most used phenomenological homogenization methods. The mechanical behavior of the composite is obtained as the homogenized results of the mechanical properties of the components assuming an isostrain compatibility equation among them. Another important formulation, based on a phenomenological homogenization, that accounts for a more general topological distribution of the components in the composite is the Serial/Parallel (SP) mixing theory. This theory, developed by [2] [3], distinguishes two behaviors of the composite depending on the alignment of the fiber on the composite. Iso-strain and iso-stress conditions are applied in the fiber direction, and in the orthogonal direction, respectively. The serial-

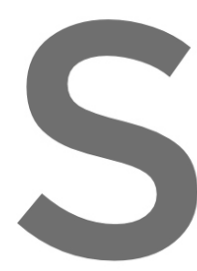
parallel theory obtains the response of the composite assuming certain iso-stress and

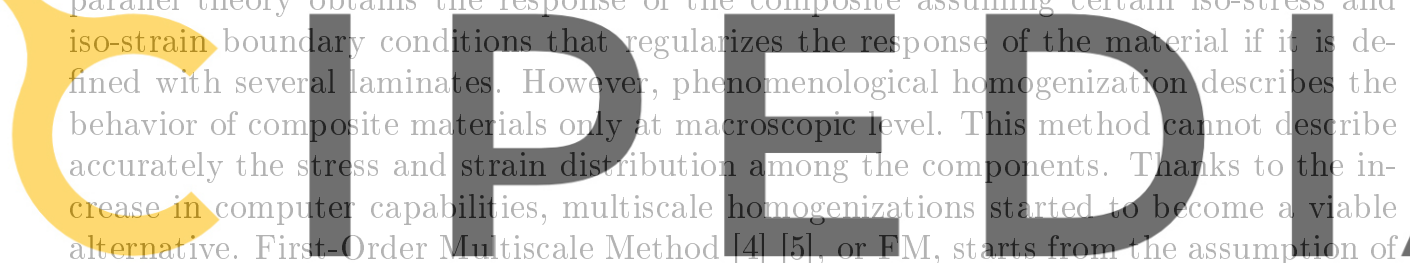
the scale separation from the macro and the microstructure [6] and analyzes the behavior

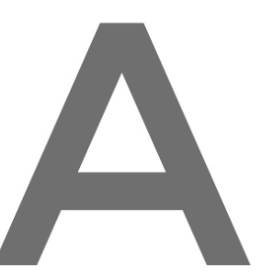

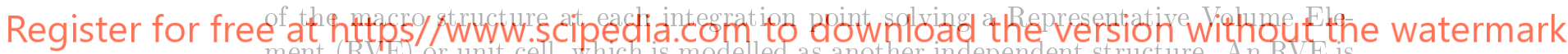

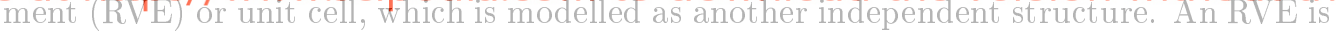
a geometrical representation of the microstructure, subjected to the macroscopic deformation gradient. Complex RVE, with linear and non-linear behaviors, could be modelied with FM method as it is studied by [7] [8] [9] [10] [11]. Of course, this methodology has a high computational cost since the microstructure shall be calculated for each integration point of the macroscale. Indeed, it will replace the constitutive law representing the material. Several model reductions were developed for the optimization of that type of analysis like Response Surface Models (RSM) [12], proper orthogonal decomposition (POD) [6] or others [13] [14]. When considering the problem of localized deformations, we can observe that in most of the cases the fracture only involves a very small area of the structure. This means that most of the material is still in the linear-elastic regime and does not require the RVE. In particular, Otero et al. [14] use a non-linear activation function that is defined considering the maximum level of an elastic energy density that can be applied to the RVE before its failure. Despite the high computational effort obtained with this procedure, with this method any changes at the macroscale level require the re-computation of the non-linear activation function even when using the same RVE. Another approach that can be used to optimize the computational cost of multiscale analyses consist on just determining the failure threshold of the structure. In this case the full multiscale analysis is replaced by a linear calculation using the obtained mechanical 
homogenized properties of the RVE. Then, in a post-process analysis, the most critical RVE are studied, i.e. those with larger elastic stresses, subjecting them to the same loads of the macrostructure.

The purpose of this work is to define a computationally efficient multiscale procedure able to predict the mechanical non-linear response of composite materials. This will be achieved, using an RVE Data Base (DB) calculated a-priori. Through the definitions of an equivalent damage parameter $\left(d_{e q}\right)$, function of the global stress at the microscale, a series of strain controlled virtual tests of the RVE are performed storing in the DB the homogenized stress and strain state reached at certain levels of $d_{e q}$. Afterwards, the solution of the macroscale structure via Classical First-Order Multiscale Method is replaced by the interpolation of the stored data.

The first proposed method, named Discrete Threshold Surface definition (DMTS), will provide the generation of the RVE in the non-linear part of the structure, while the second one, named Discrete Multiscale Constitutive Model (DMCM), is completely offline and uses only the stress information stored in the DB. Special attention has been paid on creations and validation of the Data Base followed by the study of a complete composite structure comparing the speedup obtained with both methods.

In the first part of the paper we give a brief description of the First-Order Multiscale Methods. Then we report the algorithm and the formulation for both proposed techniques with special attention on the Data Base creations and implementation. After-

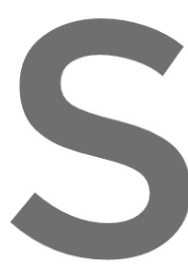
wards, as validation, we Finally, we study a complet both cases.

1. Classical First-Order
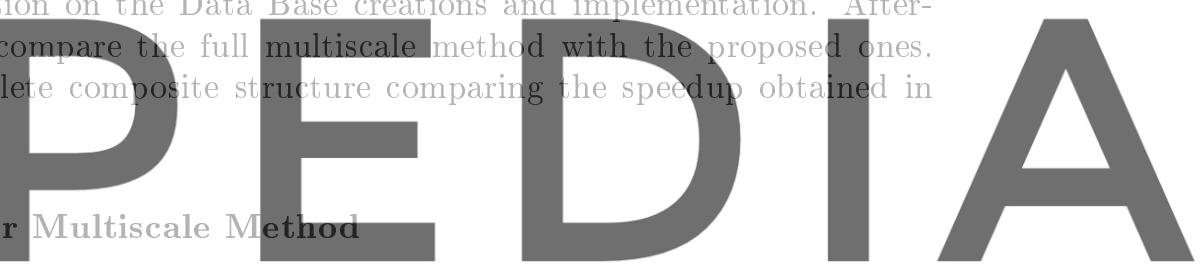

One of the most popular multiscale methods is the first-order homogenization. In this fine the boundary conditons, applied on a Representative Volume Element (RVE), to solve the Boundary Value Problem (BVP) at the microscale. The basic principles of homogenization method were provided by Suquet [5] to obtain the constitutive equation for the homogenized properties of a heterogeneous material. The unit cell is defined as a microscopic subregion that is representative of the entire microstructure in an average sense. The RVE is employed to obtain the effective properties for the homogenized material because it is assumed that it must contain a sufficient number of heterogeneities [15] [16]. The solution of the problem at the microscale, under such conditions, acts as an equivalent constitutive law for the macroscale, and it provides material stiffness and stresses as the volume average of the microscopic ones. This equivalent constitutive law is used in all the integration points of the macroscopic model to obtain the global response of the structure. When dealing with nonlinear microstructures, it will lead to an iterative procedure in which the RVE must be solved for different boundary conditions until both scales reach equilibrium, ensuring consistency between the microand macroscale solutions. The first-order homogenization technique developed assumes a scale separation between the macro and the microscale. This is, the characteristic length of the microscale should be much smaller than the length of the macroscale elements, $L: l<<L[6]$. The main steps of a classical FM technique can be resumed in the Figure [1]. On each integration point of the discretized macroscale domain, the macroscopic 
strain tensor provides the input variables for the microscale domain. Then, the solution of the microscopic behavior of the RVE provides the macroscopic output and properties of the equivalent homogeneous medium.

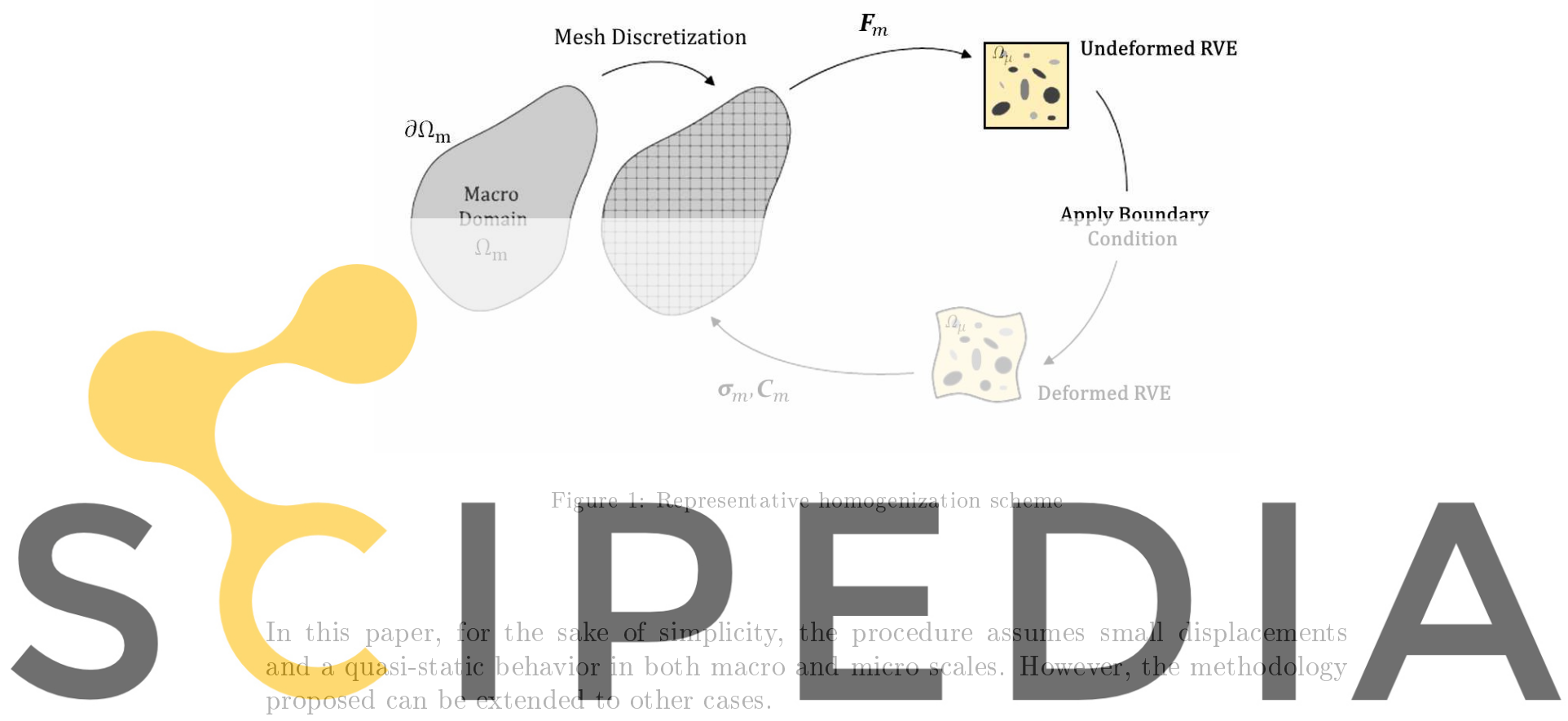

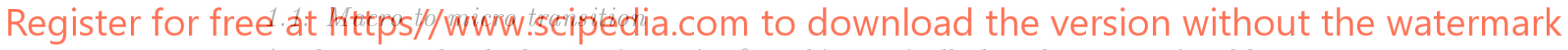

At the macro level, the starting point for a kinematically based computational homoge-

nization method is the assumption that the mechanical strain tensor, $\varepsilon_{m}$, at each point

of the macroscale domain, $\Omega_{m}$ (where the position is defined through the vector $\boldsymbol{x}_{m}$ ), and at a certain instant $t$ can be obtained as the volume average of the microscopic mechanical strain field, $\varepsilon_{\mu}$, defined at each point of the microscale domain, $\Omega_{\mu}$ (where the position is defined through the vector $\boldsymbol{x}_{\mu}$ ), and at the same instant $t$ as:

$$
\varepsilon_{m}\left(\boldsymbol{x}_{m}, t\right)=\frac{1}{V_{\mu}} \int_{\Omega_{\mu}} \varepsilon_{\mu}\left(\boldsymbol{x}_{\mu}, t\right) d V
$$

From the Eq. [1] the microscopic strain field can be expressed as the symmetric gradient of the microscopic displacement field, $\boldsymbol{u}_{\mu}=\left(u_{\mu}^{x}, u_{\mu}^{y}\right)$ :

$$
\boldsymbol{\varepsilon}_{m}\left(\boldsymbol{x}_{m}, t\right)=\frac{1}{V_{\mu}} \int_{\Omega_{\mu}} \nabla^{s} \boldsymbol{u}_{\mu} d V
$$

Or using the Gauss theorem:

$$
\boldsymbol{\varepsilon}_{m}\left(\boldsymbol{x}_{m}, t\right)=\frac{1}{V_{\mu}} \int_{\partial \Omega_{\mu}} \boldsymbol{u}_{\mu} \otimes_{s} \boldsymbol{n} d A
$$


Where $\boldsymbol{n}$ is the outward unit normal field on the RVE boundary $\delta \Omega_{\mu}$.

Without loss of generality, we can decompose the microscale displacement as:

$$
\boldsymbol{u}_{\mu}\left(\boldsymbol{x}_{\mu}, t\right)=\boldsymbol{\varepsilon}_{m}\left(\boldsymbol{x}_{m}, t\right) \cdot\left(\boldsymbol{x}_{\mu}-\boldsymbol{x}_{g}\right)+\tilde{\boldsymbol{u}}_{\mu}\left(\boldsymbol{x}_{\mu}, t\right)
$$

where $\boldsymbol{u}_{\mu}\left(\boldsymbol{x}_{\mu}, t\right)$ and $\tilde{\boldsymbol{u}}_{\mu}$ are respectively the constant and fluctuation displacements with respect to the average fields $\boldsymbol{x}_{g}$ (the centroid of the microscale) at each instant $t$.

In the same way the microscopic strain can be divided in two parts, a constant one from the macroscopic scale $\left(\varepsilon_{m}\right)$ and the contribution of fluctuation $(\tilde{\varepsilon} \mu)$.

$$
\varepsilon_{\mu}\left(\boldsymbol{x}_{\mu}, t\right)=\varepsilon_{m}\left(\boldsymbol{x}_{m}, t\right)+\tilde{\boldsymbol{\varepsilon}}_{\mu}\left(\boldsymbol{x}_{\mu}, t\right)
$$

Moreover, the microscopic position vector $\boldsymbol{x}_{\mu}$ does not appear explicitly in the microstructural strain tensor expression (see Eq. [5]). Consequently, this variable does not appear in the microstructural stress tensor either. Therefore, the periodic microstructure around the macro point $\boldsymbol{x}_{m}$ does not have to be modeled with its exact dimensions. A non-dimensional RVE with the internal distribution and volume fractions of the simple materials is enough to obtain the microscopic strain and stress fields. This is one of the principal advantages of this first-order homogenization approach compared to other multiscale high-order approaches [14]. On the other hand, it can be observed that the kinematically admissible displacement fluctuation option used to satisfy the boundary
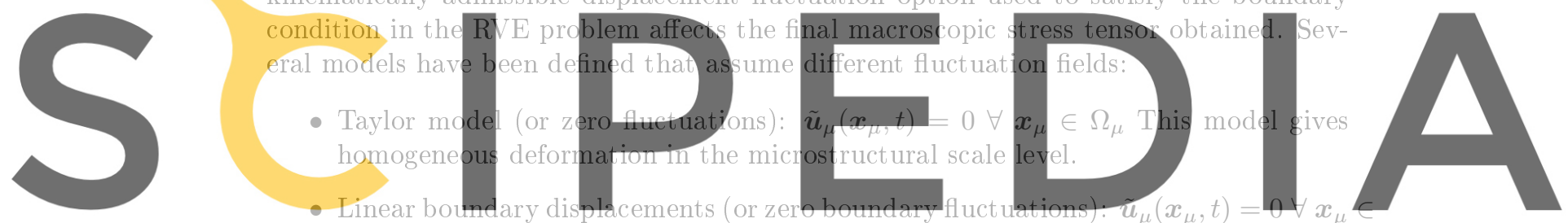

$\partial \Omega_{\mu}$. The deformation of the RVE boundary domain for this class are fully pre-

Register for free at lfetepss//www.scipedia.com to download the version without the watermark

- Periodic boundary fluctuations: $\tilde{u}_{\mu}\left(x_{\mu}^{+}, t\right)=\tilde{u}_{\mu}\left(x_{\mu}^{-}, t\right) \forall \operatorname{pair}\left\{x_{\mu}^{+}, x_{\mu}^{-}\right\} \in \partial \Omega_{\mu}$ The key kinematical constraint for this class is that the displacement fluctuation must be periodic on the different faces of the RVE.

- Minimal constraint (or uniform boundary traction): In this constraint the nontrivial solution of $\int_{\partial \Omega_{\mu}} \boldsymbol{u}_{\mu} \otimes_{s} \boldsymbol{n} d A$ is obtained.

For the displacement fields, we use periodic boundary conditions since they generally provide an intermediate and more exact response compared to other type of boundary conditions, as it is described in [16] [17] [18] [19] [14].

\subsection{Micro to macro transition}

Following the solution of the BVP [20] we get the homogenized macroscopic stress tensor. In addition, we can obtain the homogenized constitutive tensor. The homogenized macroscopic stress tensor can be obtained as the microscopic stress field of the RVE averaged on the volume as [6] [21] [22]

$$
\boldsymbol{\sigma}_{m}=\frac{1}{V_{\mu}} \int_{\Omega_{\mu}} \boldsymbol{\sigma}_{\mu}\left(\boldsymbol{x}_{\mu}, t\right) d V
$$




\subsection{Material homogenized properties}

The macroscopic constitutive relation defined by the homogenized properties of the RVE can be obtained after the solution of the microscale BVP. Assuming the equilibrium of the microscale expressed as:

$$
\int_{\Omega_{\mu}} \boldsymbol{\sigma}_{\mu}\left(\boldsymbol{x}_{\mu}, t\right): \nabla^{s} \tilde{\boldsymbol{u}}_{\mu} d V=0
$$

As is described in [23] and [14], the homogenized constitutive tensor $C^{H}$ can be defined as:

$$
C^{H}=\frac{1}{V_{\mu}} \int_{\Omega_{\mu}} C_{\mu} d V
$$

where $\boldsymbol{C}_{\mu}$ is the material constitutive tensor of the RVE. The evaluation of the homogenized constitutive tensor is performed with a perturbation method, also see[8] [14]. For each column $j$ of the constitutive tensor, a small strain perturbation $\left(\delta \tilde{\varepsilon}_{j}\right)$ is applied to the RVE in order to obtain, along with Eq. [7], a perturbed stress tensor $\left(\delta \tilde{\sigma}_{j}\right)$. The $j$ columns of the homogenized constitutive tensor can be obtained as:
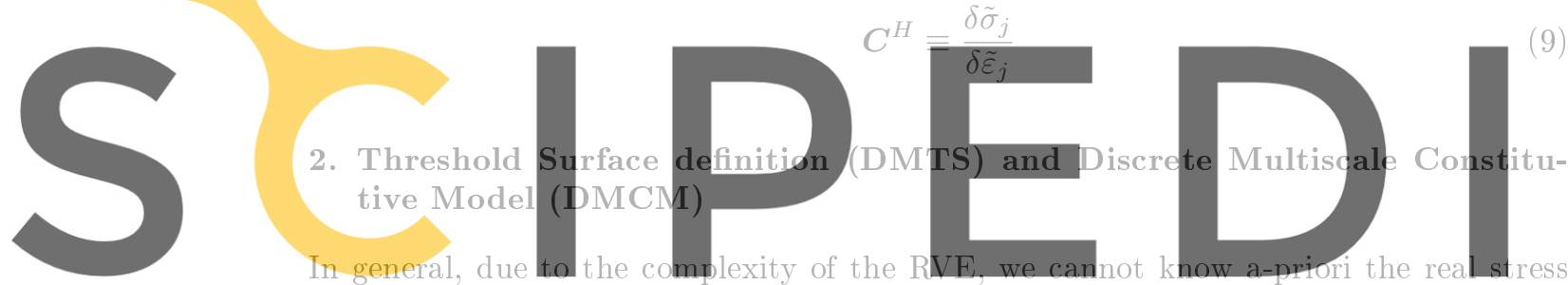

behavior of the microscale. To overcome this problem, we will study the strain space

The purpose of this paper is to provide two multiscale optimization techniques, the

Discrete Miuitiscaie Threshoid Surface (DMIS) and the Discrete Miultiscale Constitutive Model (DMCM), directly derived from the classical first order multiscale theory (FM), using periodic boundary condition as reference for future comparisons.

Considering these two methods, the main aim is to construct a strain collector Data Base based on multiple simulations of the RVE able to describe the microscale behavior of the material.

The DMTS method will describe with a finite number of points, stored in the strain collector Data Base, the boundary (threshold surface) of the elastic regime. Identifying where a full RVE needs to be employed and where the homogenized elastic properties are sufficient to obtain the macroscale response.

As an extension of the DMTS, the DMCM method aims to decouple the macroscale analysis from the FM, interpolating multiple surfaces (also stored in the Strain Data Base) that describes both linear and non-linear regime.

Below, the authors will describe in detail all the aspects of both methods, providing examples and algorithms used for the numerical implementation. 


\subsection{Strain Data Base definition}

The failure surface defined by the DMTS model, as well as the damage surfaces of the DMCM model, are defined as a function of the applied strain on the composite. These strains, defined as strain histories, are in the multidimensional spaces $\left(\varepsilon_{x x}, \varepsilon_{y y}, \varepsilon_{x y}\right)$ for $2 \mathrm{D}$ or $\left(\varepsilon_{x x}, \varepsilon_{y y}, \varepsilon_{z z}, \varepsilon_{x y}, \varepsilon_{y z}, \varepsilon_{x z}\right)$ for $3 \mathrm{D}$ mechanical problems. To make accessible this information during the analysis we will store the strain histories in a Homogenized Strain Data Base. In this section, for simplicity, we will only describe the $2 \mathrm{D}$ case, but it is perfectly extendable to $3 \mathrm{D}$.

To obtain a complete definition of the microscale behavior we will impose as strain histories the value of equispaced points projected on the sphere of unitary radius centered in the origin of the axes $\left(\varepsilon_{x x}, \varepsilon_{y y}, \varepsilon_{x y}\right)$. We can uniquely define these points in $3 \mathrm{D}$ spherical coordinates system as the combination of three parameters $(\theta, \varphi, \lambda)$, as we can see in the Figure [2](a). Where the angles $\theta$ and $\varphi$ represents the direction of the strain loads that we applied to the microscale and $\lambda$ is the unitary strain intensity. Then we can obtain the components of the strain vector in cartesian coordinates as:
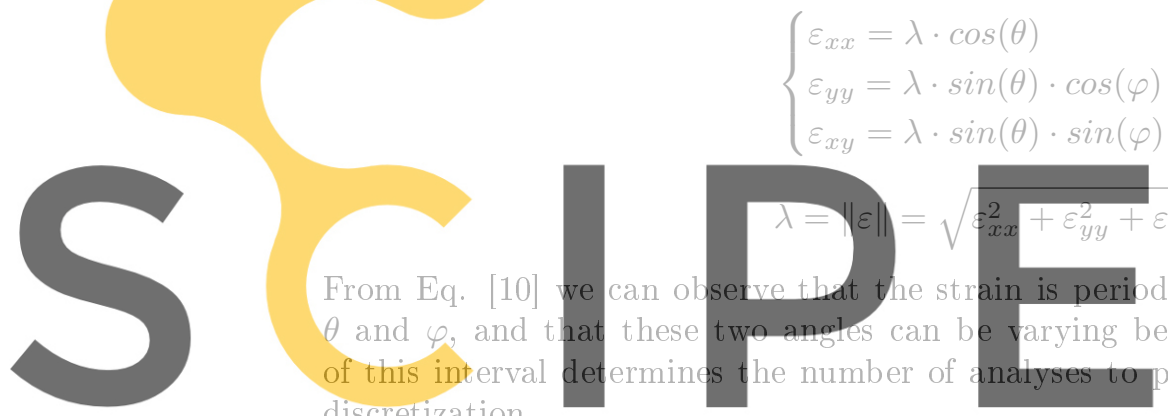

$(10)$

Register for free at https//www.scipedia.com to download the version without the watermark
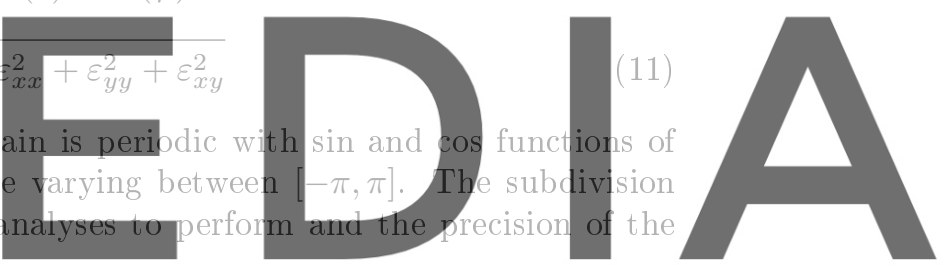

discretization

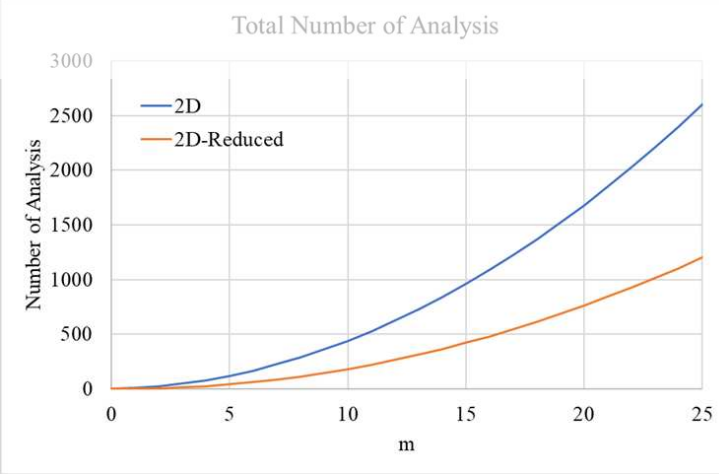

Figure 2: (a)3D Strain Space, (b) Total number of analysis for 2D and 2D Reduced case

Introducing the parameter $m \in[1, \infty)$ as subdivision of the interval $[0, \pi]$ we can uniquely define the strain direction with a pair of integer parameters $\in[-m, m]$, that we will call 
tag. With this method, each point is equally spaced from the other and the angles $\theta$ and $\varphi$ vary between $\left[\frac{-\pi}{m}, \frac{\pi}{m}\right]$. In that way, we can observe that the total amount of analyses is $(2 \cdot m+1)^{2}$. Considering the superposition of the same resulting strain directions the amount of strain histories can be reduced to $(2) \cdot(m-1)+2$. Despite this reduction, we will remark that using a high value of $m$ implies an exponential increasing of the analyses, see Figure [2](b).

Once the strain loading directions are computed, we apply them to the RVE doing a classical First Order Multiscale Analysis, as described in the section 1. As our interest lays in the RVE performance, to reduce the computational cost this analysis is conducted in a macroscale triangular FE element with a single gauss point, and in which the strains are imposed as fixed displacement to the nodes. In this case, all the degrees of freedom of the triangle are fixed and for each $n$ time step we will solve the microscale problem, evaluating the homogenized stress and constitutive tensor. With these information we can determine the corresponding value of equivalent damage at each time step.

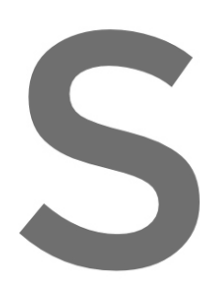

2. Equivalent damage definition

During a classical full multiscale analysis, the RVE is solved at each time step even if the linear elastic limit of the material is not achieved. In terms of computational cost, this is highly inefficient. The two procedures proposed in this work will optimize this

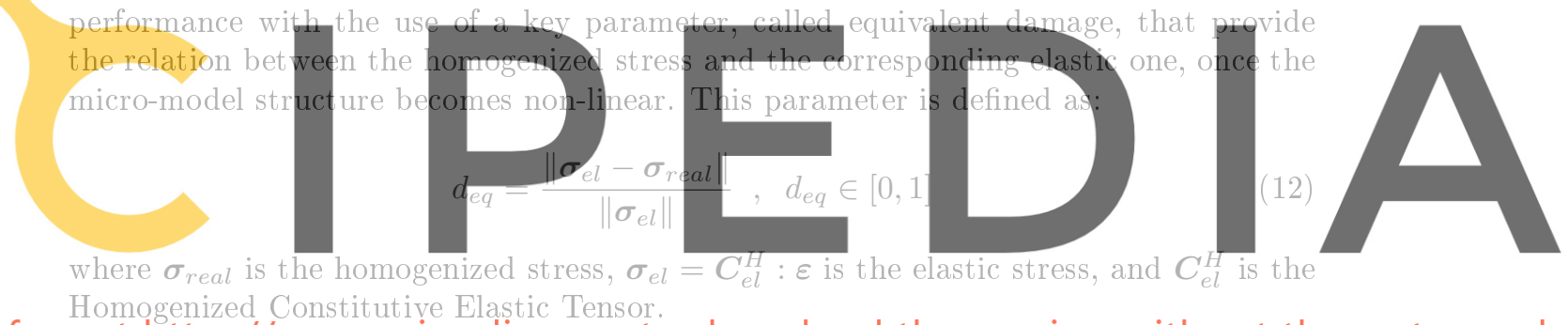
Homogenized Constitutive Elastic Tensor.

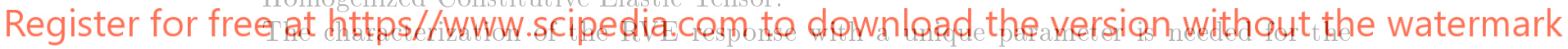
interpolation of the Data Base DB information. In this way, we can obtain the stress flux surface at certain equivalent damage level and reconstruct the RVE behavior for any strain directions interpolating the stored homogenized stress data. To speed up the Data Base look-up and the parsing process, the information is stored in json format [24] that provides a flexible, more compact, easier to parse and more human readable text.

\subsection{Discrete Multiscale Threshold Surface}

In this work we propose to define the linear elastic threshold of the microstructure when it reaches a equivalent damage, $d_{e q}$, equal to 0.1 . Therefore, the Discrete Multiscale Threshold Surface is represented by the corresponding stress at each tag studied. The DMTS Data Base structure in json format provides a list of tags and corresponding stress-strain vectors as proposed in Figure [3]: 
1

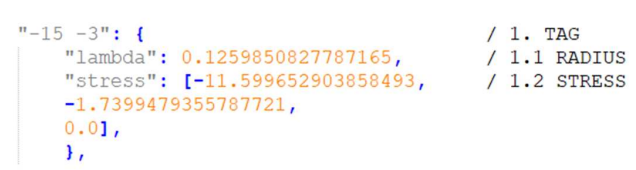

Figure 3: Json Data Base Format for DMTS

To better understand the obtained Data Base, a graphical 3D visualization of DMTS stresses in the strain space is proposed below for Tension-Compression damage material used in section 4 and well explained in [25]
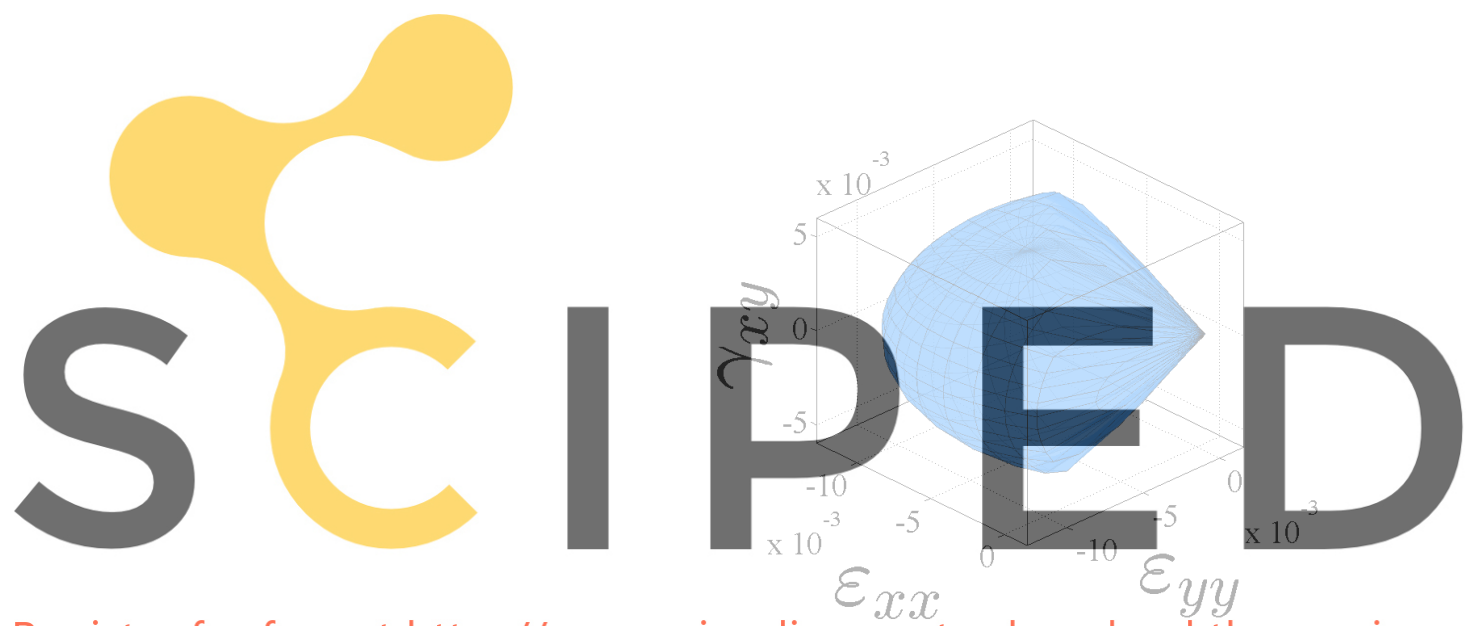

Register for free at https//www.scipedia.com to download the version without the watermark

Figure 4: 3D visualization of DMTS for Tension-Compression Damage Law

Figure [5] shows a 3D visualtionation of a portion of the DMTS surface. In this figure point $\mathrm{E}$ corresponds to intersection of the elastic limit surface with the stress and strain tensor applied on the macroscale structure. The points $A, B, C, D$ correspond to the nearest tags to point $\mathrm{E}$, which are used to interpolate the results. 

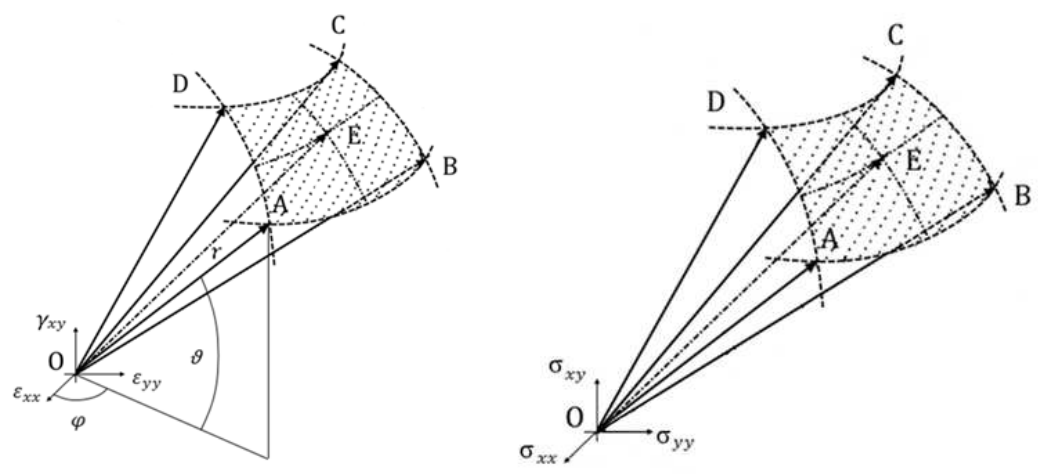

(a)

(b)

Figure 5: Graphical representation of (a)Strain Space and (b) Stress Space for DMTS

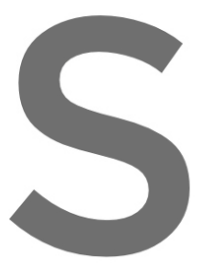

The DMTS proced shown in Figure there are some elements of is no longer valid. will be used to ob whole procedure is
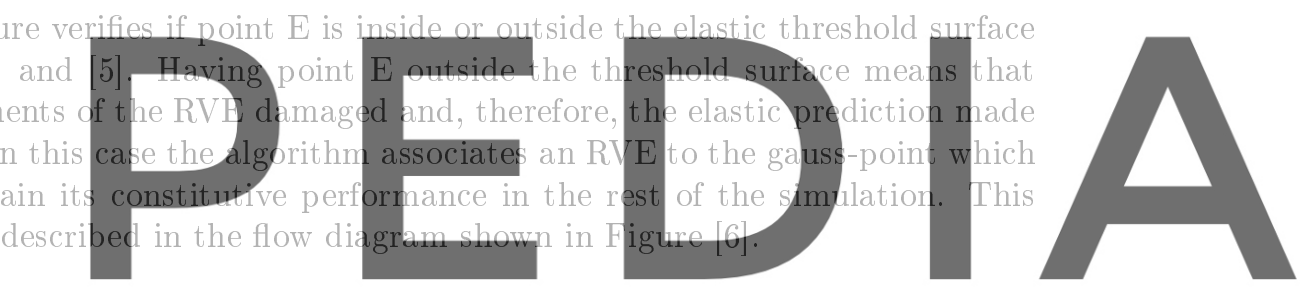

Register for free at https//www.scipedia.com to download the version without the watermark 


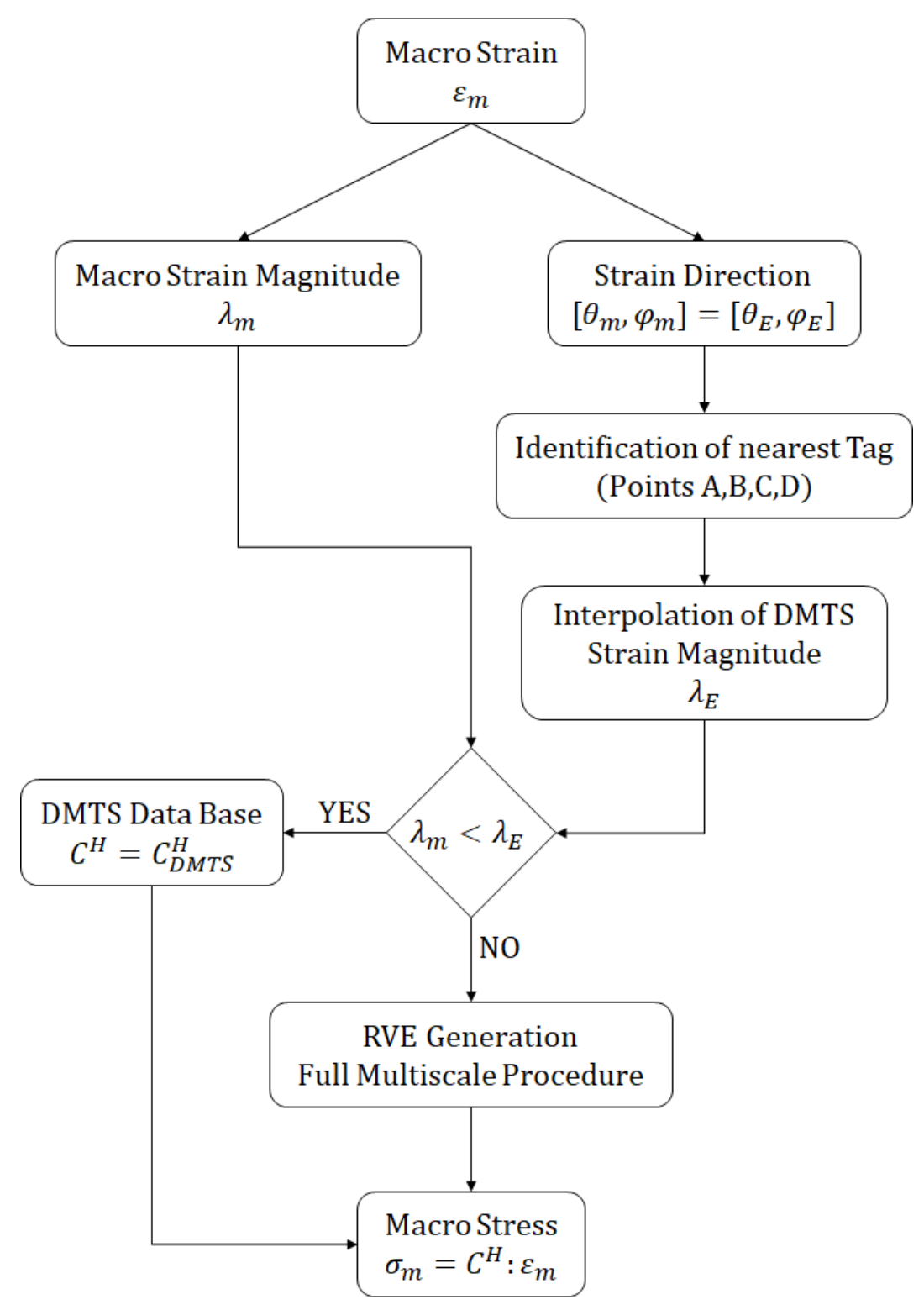

Figure 6: DMTS Algorithm

\subsection{Discrete Multiscale Constitutive Model}

The natural evolution of the DMTS model previously presented is the second method proposed in this work. Indeed, the DMCM model stores the linear and non-linear performance of the RVE behavior in the material Data Base. the stress-strain response related 
to the equivalent damage parameter defined previously. Instead of only one value, corresponding to the elastic threshold, the DMCM approach computes and stores multiple surfaces: the elastic threshold one and the ones corresponding to the non-linear behavior, which are discretized with a series of equivalent damage levels ranging from 0 to 1 . A finite number of surfaces are computed for the RVE, corresponding to a user-defined number of damage levels. To obtain the strain and stress tensors associated to a given value of the damage parameter, the strain increment applied to the RVE is iteratively adapted, for each strain direction (tag), until the correct damage value is obtained. Having stored the RVE performance in the linear and non-linear range, the FM analysis is no longer needed, and the mechanical response of the macro-structure can be obtained from the DB. Indeed, an additional interpolation method over the stored threshold surfaces could be performed to predict the behavior of the macrostructure at each integration point.

During the macroscale analysis, the algorithm evaluates the strain direction only at the current time step without information about any previous strain orientation or magnitude. In case of changes in the load or coupling of failure phenomena, a misalignment between the real loading path of the RVE and the DMCM solution is possible. Nevertheless, the validation examples proposed in section 4 provides a good correlation between the Full Multiscale Method and the DMCM Method, making the error affordable.

Figure [7] shows a graphical representation, in the strain and stress space, of the DMCM procedure.

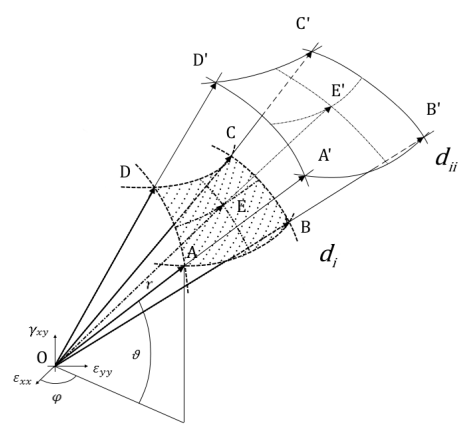

(a)

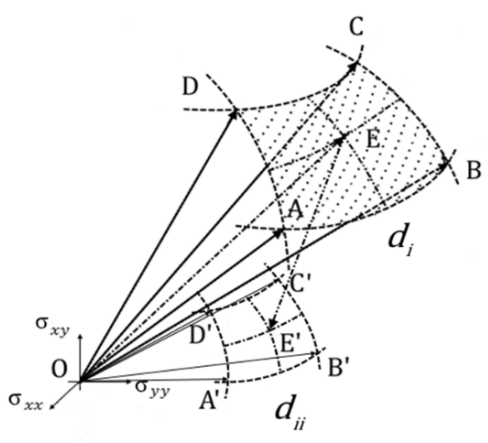

(b)

Figure 7: Graphical representation of (a)Strain Space and (b) Stress Space Evolution for DMCM

Points $E$ and $E^{\prime}$ represent the intersection of the direction of the stress and strain with the surfaces at damage $i$ and $i i$. Points $A, B, C, D$ and $A^{\prime}, B^{\prime}, C^{\prime}, D^{\prime}$ corresponds to the nearest tag that is surrounding this direction for the different level of damage. The value of strain and stress for points $E$ and $E^{\prime}$ were determined by interpolating the data from the DB of the nearest tag for each level of damage. In this way we can reconstruct the stress strain response of the RVE for any possible direction in the strain space. The DMCM algorithm is resumed in Figure [8]: 


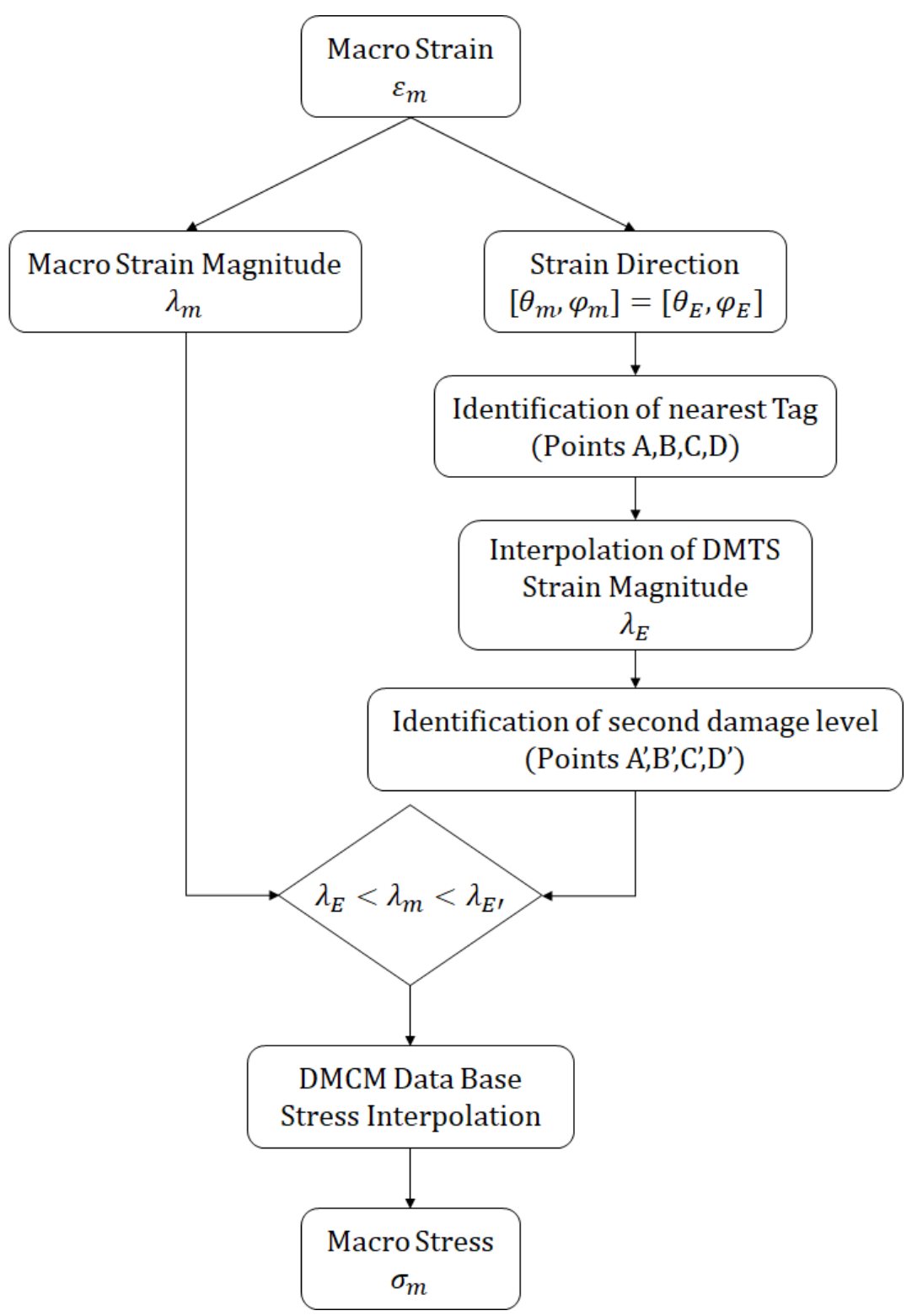

Figure 8: DMCM Algorithm

It is important to remark that, creating the DB with strain-controlled analysis, the reference surfaces in the strain space are convex. Considering advanced materials as reinforced composites, the high dependence on the RVE geometry and the constitutive performance of its constituent materials provide a global response comparable to a complex structure, mathematically more complex than a classical homogeneous material. This complexity 
reflects to as well complex stress threshold surfaces.

In the same way as the DMTS, the DMCM Data Base was stored in json format [24].

1

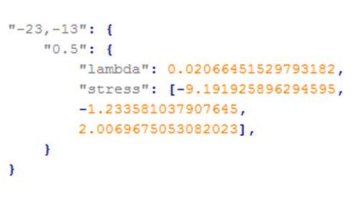

$\begin{array}{ll}1.1 & \text { TAG } \\ 1.1 & \text { DAMAGE } \\ 1.1 .1 & \text { RADIUS } \\ 1.1 .2 & \text { STRESS }\end{array}$

Figure 9: Json Data Base Format for DMCM

The DB scheme provides at first level the tag. Then, for each tag, we will store the information of $\sigma$ and $\lambda$ for different values of equivalent damage analyzed.

Considering the number of analysis needed to create the stress-strain DB, which is proportional to the discretization parameter $\mathrm{m}$, it is obvious that as long as the number of integration points at the macroscale level is higher than the number of tags, the advantages of the proposed method increases. Moreover, as we can see in the Figure [10], where the numbers of RVEs simulations in case of DMCM is the total number of analyses needed to create the stress-strain database. The author will remark that this number is independent from the number of damage levels because for each analysis multiple values can be stored. Indeed, in case of FM, as explained in section 1, the microscale structure needs to be solved for each integration points of the macroscale.

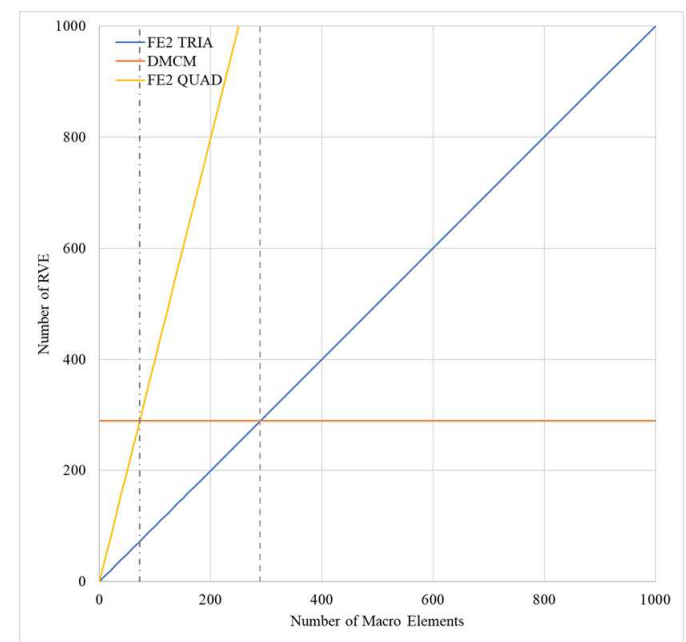

Figure 10: Number of RVEs simulations in a 2D multiscale analysis for FM (first order triangular and quadrilateral elements) and DMCM (discretization parameter $m=8$ ) 


\subsection{Interpolation Methods}

In order to determine if the stresses in the macro-model are below the threshold surface or to know the equivalent damage parameter associated to the stress state, in the DMTS and DMCM models respectively, we need to use an interpolation technique because the most frequent scenario is that the strains associated with the stress analyzed do not correspond to any of the tags previously calculated. The interpolation methods implemented in DMTS and DMCM technique are:

- Nearest-neighbor: This method provides as output the value of the nearest point without considering other neighboring points.

- Linear: this type of interpolation needs to know the values of the nearest 4 points in order to perform a linear interpolation over each dimension.

- Cubic interpolation: in case of $2 \mathrm{D}$ interpolation this method required the values of the nearest 16 points in the input data.

A comparison of the tree methods has been made for a well-known J2 material. In Figure [11] is reported the elastic boundary in the strain space $\left(\varepsilon_{x x}, \varepsilon_{y y}\right)$ for the $2 \mathrm{D}$ case. The black line represents the conjunction of the reference tags, while the different interpolation methods are colored in green for the nearest-point method, in blue for the linear method and in red for the cubic one. The authors observe that the Cubic interpolation provides the best fitting of the theoretical J2 ellipsoid. Instead, the linear interpolation tends to underestimate the interpolated magnitude due to the convexity of the surfaces under study, as has been explained in section 2.3. Finally, Figure [11] shows that the nearest neighbor approach does not provide good results, unless the known magnitudes are very closed together.

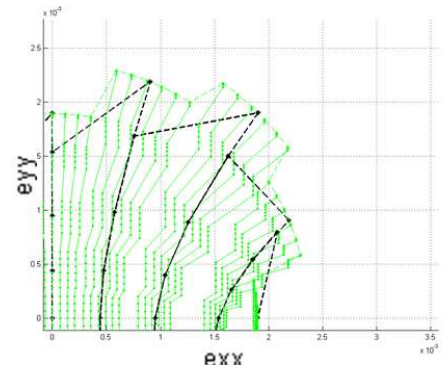

(a)

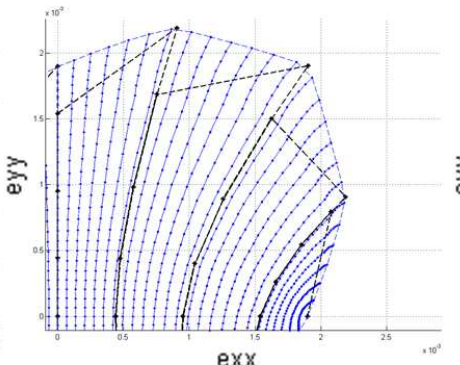

(b)

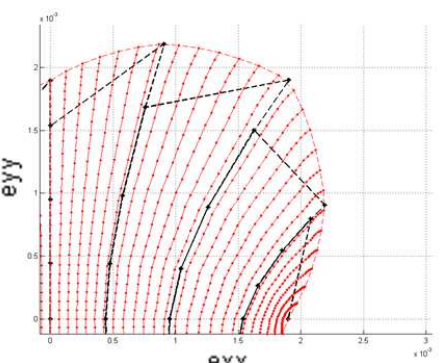

(c)

Figure 11: Comparison of Different Interpolation Techniques applied to a J2 plasticity constitutive law database (black dashed lines). Section of $\left(\varepsilon_{x x}, \varepsilon y y\right)$ plane with (a) nearest-point, (b) linear and (c) cubic interpolation method. 


\section{Data Base Regularization}

In a FEM analysis an important aspect to consider is the mesh discretization of the structure, namely the results must be independent of the mesh size. These aspects were extensively investigated in [26] [27] [28] [29], where it is possible to observe how, during the fracture process, the localization appears only on one band of the mesh. This localization, as well as the fracture energy $\left(G_{f}\right)$ dissipated in during the fracture process, must be independent of the mesh size. This is achieved by defining a volumetric fracture energy $\left(g_{f}\right)$ which is associated to the finite element and its size. Larger finite elements will dissipate less energy per unit volume, in order to achieve the same structural response, as we can see in Eq. [13]:

$$
g_{f}=\frac{W}{A} \frac{1}{l_{f}}=\frac{G_{f}}{l_{f}}
$$

Where $W$ is the total energy dissipated during the fracture process, $A$ is the fracture area, $G_{f}$ is the fracture energy per unit of crack area $A$ and $g_{f}$ is the fracture energy per unit of volume. In case of multiscale analysis preserving the mesh objectivity is not obvious and, as explained in [14] or [30], both characteristic lengths of the FE elements, at the micro $\left(l_{f, \mu}\right)$ and macro $\left(l_{f, m}\right)$ scale, need to be taken into account. In particular, Otero et al. analysis preserving the mesh objectivity is not obvious and, as explained in [14] and Petracca et al. [30] introduced a modified characteristic length, that provides a relation between the two length scales and the total length of the RVE $\left(l_{f, R V E}\right)$, in order to preserve the correct dissipation.

$$
\tilde{l}_{f}=f\left(l_{f, \mu}, l_{f, m}, l_{f, R V E}\right)
$$

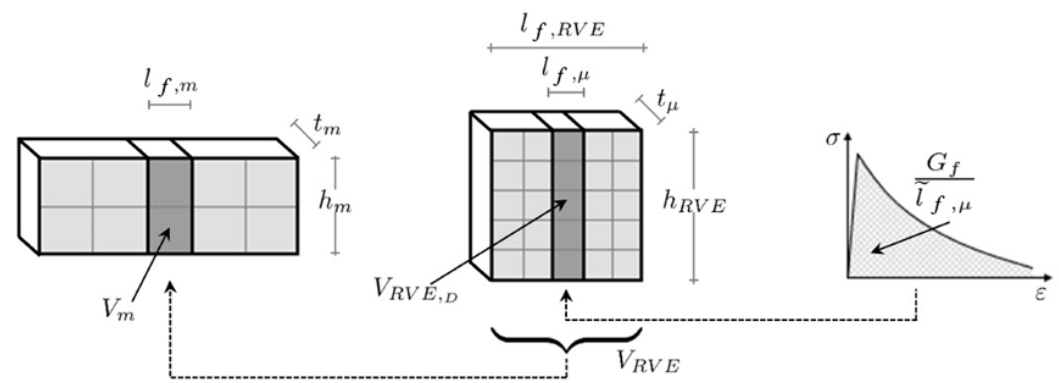

Figure 12: Fracture energy based regularization in 2-scale FEM (Petracca et al. [30])

This modified characteristic length and the macroscale dissipation were defined as follow:

$$
\begin{gathered}
W_{m}=\int_{A_{m}} g_{f} d V_{m} \\
W_{m}=g_{f} l_{f, m} h_{m} t_{m} \\
16
\end{gathered}
$$


Where, $h_{m}$ is the length and $t_{m}$ is the thickness of the crack at the macroscale as in Figure [12]. During the creation of the DB, this regularization process was adopted, where the $l_{f, m}$ is the length of the macroscale element used to calculate the material database, that from now on we will call $l_{f, D B}$. Considering the intrinsic strain dependency of the DB we will introduce a parameter $\alpha$, function of the finite element size and the global fracture energy, that will modify the stored DB strain proportionally to the mesh refinement of the macroscale. To determine $\alpha$ we will define $G_{f, D B}$ as the global fracture energy calculated as the integral of the norm of the stress, and $G_{0}$ as the elastic counter part.

$$
\begin{gathered}
G_{f}=G_{f, D B} \frac{l_{f, D B}}{l_{f, m}} \\
\alpha=\frac{G_{f}-G_{0}}{G_{f, D B}-G_{0}}-1
\end{gathered}
$$

Then, at each time step $i$ the regularized strain $\varepsilon_{i}$ is calculated as in Eq. [19]. Where $\varepsilon_{i, D B}$ is the DB strain at the step $i$ and $\varepsilon_{0}$ is the DB strain corresponding to the linear elastic boundary calculated for the DMTS method.

$$
\varepsilon_{i}=\varepsilon_{i, D B}+\alpha\left(\varepsilon_{i, D B}-\varepsilon_{0}\right)
$$

In order to validate the proposed regularization approach, three different models of the same geometry with different mesh sizes were studied, comparing the results with and without regularizations. Geometry and mechanical properties of the model are given respectively in Figure [13] and Table [1].

Table 1: Material Properties

\begin{tabular}{cccc}
\hline$E[M P a]$ & $\nu$ & $\sigma_{t}[M P a]$ & $G_{t}\left[\frac{J}{m^{2}}\right]$ \\
\hline 20000.0 & 0.15 & 2.2 & 0.012 \\
\hline
\end{tabular}



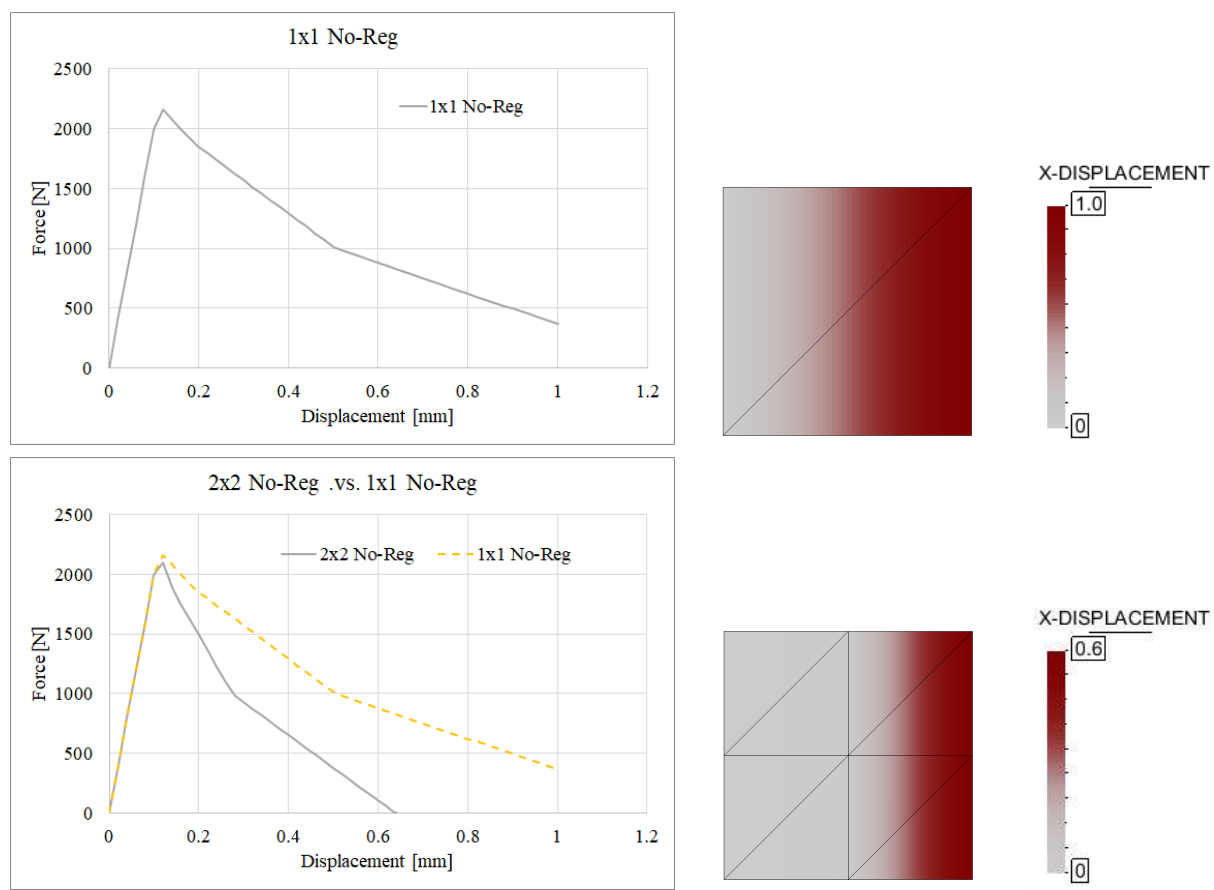

(a)
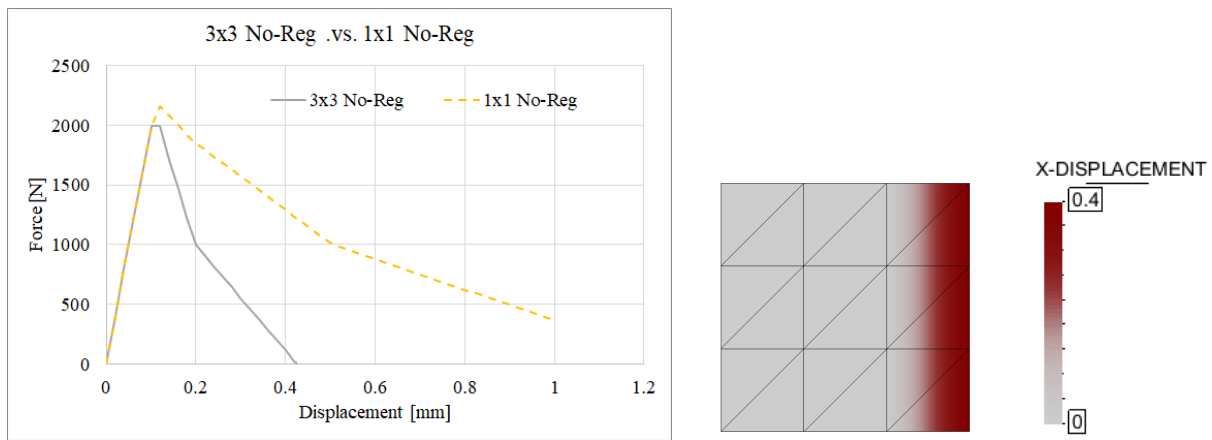

(b)

Figure 13: Comparison of Force-Displacement evolution between different mesh size for Non-Regularized Method. (a) Macro 1x1, (b) Macro 2x2, (c) Macro 3x3. The contour field represents the applied displacement.

As expected, the response is more brittle with finer meshes and using the proposed regularization technique all the analyses give the same result as we can see in the Figure [14], obtaining the objectivity respect to the macro-scale FE size. 


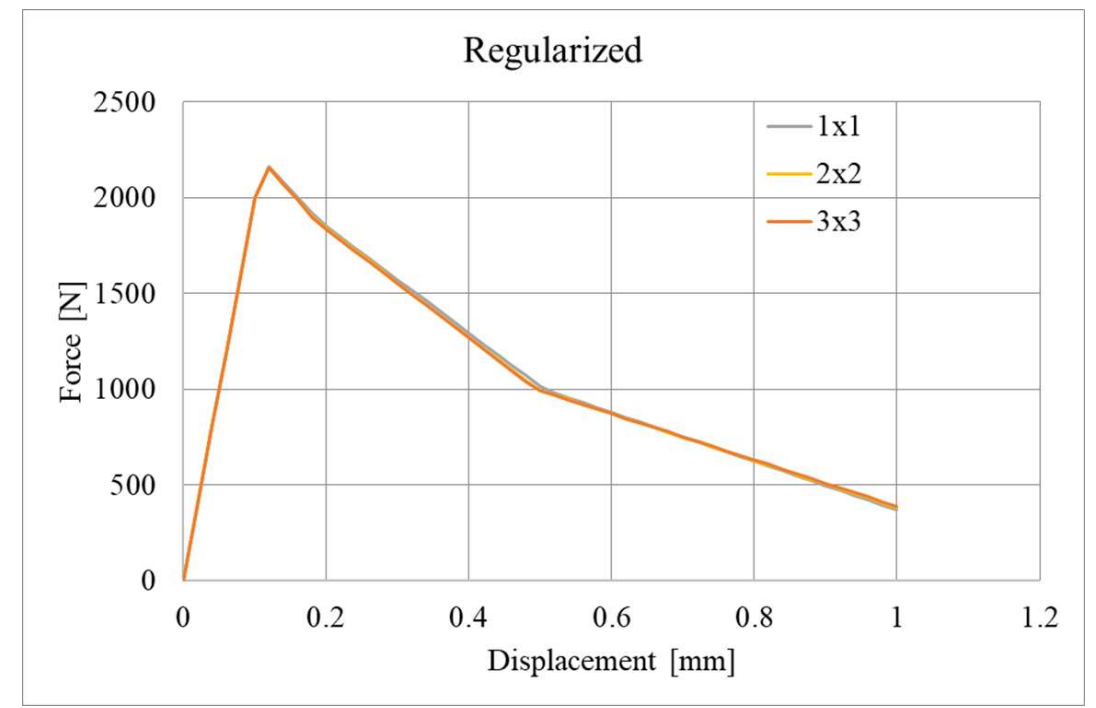

Figure 14: Force-Displacement Curve corresponded to the Regularized case.

\section{Numerical Validation}

4.1. Shear test on damage material with different threshold values for tension and compression

The first validation model that is analyzed corresponds to a shear test made on a unitary $(1 \mathrm{~m})$ square geometry. This macro-model is made with just one bulk material, which is defined using a damage law with different failure threshold for tensile and compressive stresses. This analysis can be solved without the need of a multiscale procedure. Therefore it will be used to validate the capability of the developed methods, DMTS and DMCM, to predict the material response. The macro-element is simulated with $11 \mathrm{x} 11$ small displacement quadrilateral elements; while the micro-element is simulated with a mesh of $1 \times 1$ quadrilateral element, and with a material with the damage constitutive law presented in [25], which properties are described in Table 3.

In this case, the discretization parameter used to construct the stress-strain database is $m=8$ that required a minimum of 114 analyses of the RVE. While, the levels of damage stored for each direction are [0.01, 0.05, 0.1, 0.5, 0.7, 0.8, 0.9,0.99, 0.999]. Each one of the analyses was carried out with a single standard PC equipped with an Intel Core i7-6700HQ CPU and 8 GB RAM. In Table [2] is reported the computational effort required for both DMTS and DMCM methods. 
Table 2: Computational cost for Database construction of RVE with different threshold values for tension and compression in case of DMTS and DMCM method

\begin{tabular}{ccc}
\hline Type & Time Full Database [s] & Memory Single RVE [MB] \\
\hline DMTS & 154 & 19.1 \\
DMCM & 585 & 19.1 \\
\hline
\end{tabular}

Table 3: Material Properties

\begin{tabular}{cccccc}
\hline$E[M P a]$ & $\nu$ & $\sigma_{t}[M P a]$ & $G_{t}\left[\frac{J}{m^{2}}\right]$ & $\sigma_{c}[M P a]$ & $G_{c}\left[\frac{J}{m^{2}}\right]$ \\
\hline 900.0 & 0.15 & 0.25 & 0.016 & 10.5 & 40.0 \\
\hline
\end{tabular}

Figure [15] shows a 3D representation of the surface obtained from the RVE for a equivalent damage parmater $d_{e q}=0.1$, which will be used as the failure threshold surface in the simulation. This figure also shows the cuts of this surface with planes $\left(\varepsilon_{x x}, \varepsilon_{y y}\right)$ $\left(\varepsilon_{x x}, G_{x y}\right)$ and $\left(\varepsilon_{y y}, G_{x y}\right)$. Figure [16] shows the intersection of the calculated sufraces with plane $\left(\varepsilon_{x x}, \varepsilon_{y y}\right)$ for equivalent damage values of $d_{e q}=0.1,0.5,0.9$. 


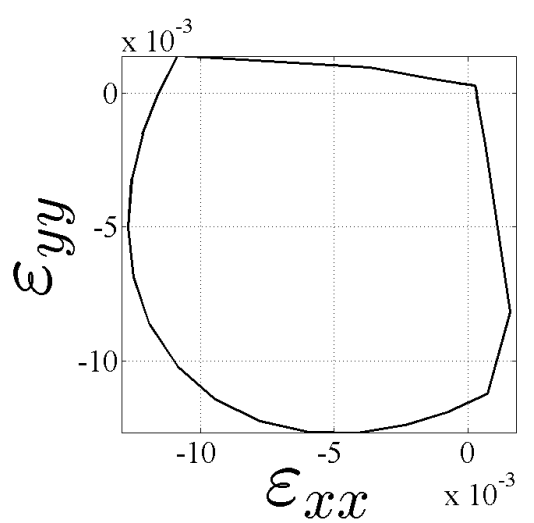

(a)

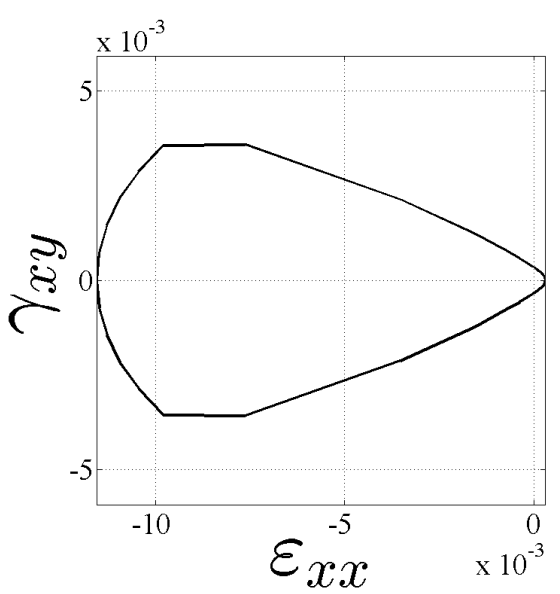

(c)

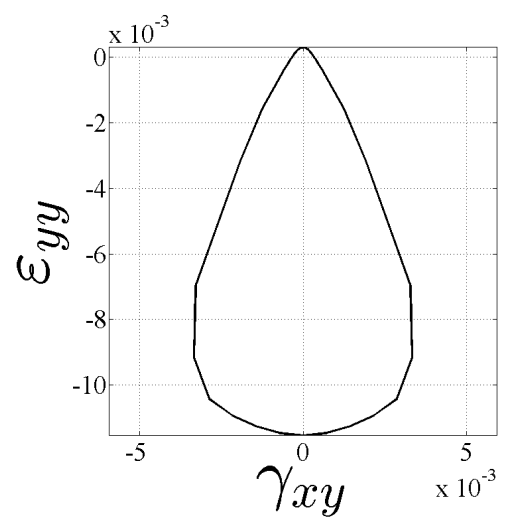

(b)

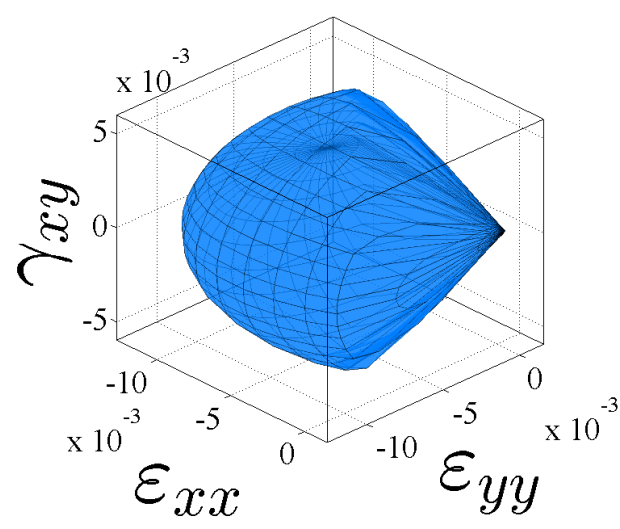

(d)

Figure 15: Elastic threshold surface for damage material with different threshold values for tension and compression 


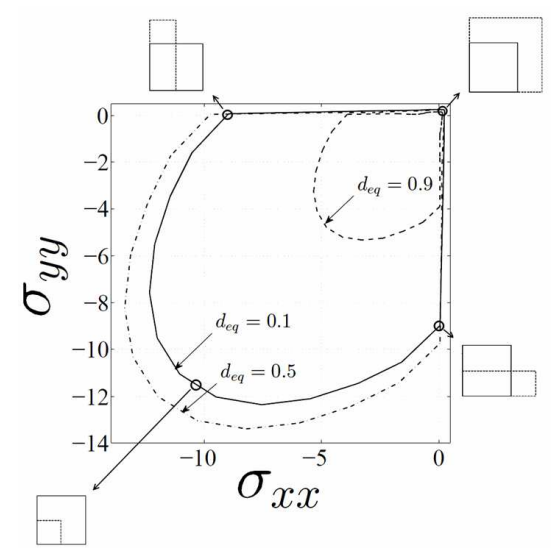

(a)

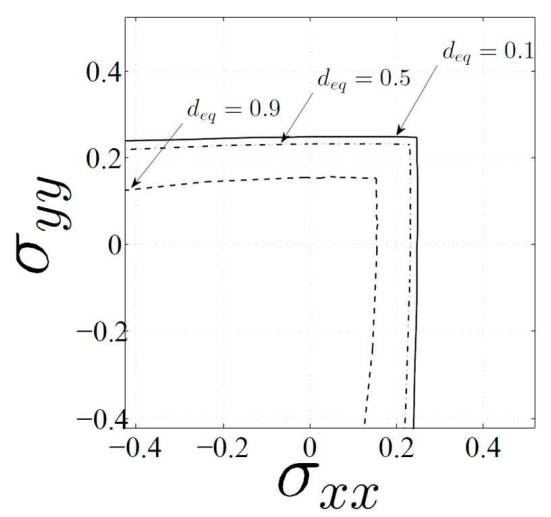

(b)

Figure 16: Threshold surface evolution for damage material with different threshold values for tension and compression

As expected, observing the stress space evolution, the surfaces become smaller with the increase of the damage parameter. In the compression zone, due to an initial hardening, the stress for $d_{e q}=0.5$ is bigger than the previous one at $d_{e q}=0.1$.

Once having analyzed the RVE in all possible directions and constructed its associated $\mathrm{DB}$, it is possible to use it to conduct macro-analysis of a structural component. As mentioned previously, current validation will be made on a square specimen subjected to a shear load.

The DMTS procedure uses the information stored in the database only to verify if the material has exceeded its elastic threshold. In such case, it will be defined an RVE associated to that material point and its mechanical performance will be obtained from the RVE during the rest of the analysis. On the other hand, the elements that are still in the linear regime do not need the RVE generation. If the analysis is conducted with the DMCM procedure, the response of the macro-scale is obtained entirely from the RVE response previously computed and stored in the DB. Therefore, the multiscale analysis is conducted only at the macro-scale level, with the obvious advantages in term of memory and time.

In order to validate properly the DMTS and the DMCM procedures proposed, this simulation has been also analyzed with a full multiscale scheme (FM). The results from this analysis will be considered reference values and will show the computational improvements obtained with the proposed methodologies. 


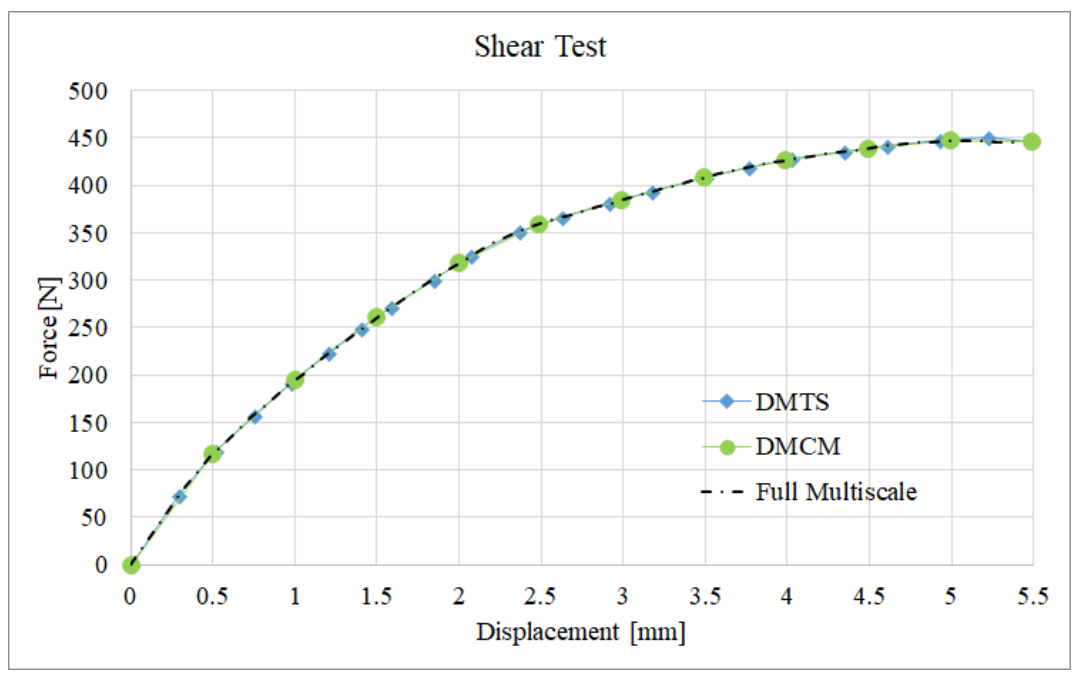

Figure 17: Force-Displacement result for shear test

Figure [17] shows that the global response of the structure, in both linear and non-linear ranges, is identical independently of the numerical approach considered, which proves the validity of the DMTS and DMCM procedures for conducting multiscale analysis of structures. Both procedures not only provide the same structural performance, but they do it with a substantially smaller computational cost, as it is shown by the values of Table [4]. In this example, the DMCM procedure reduces the computational time to a third and the computational cost to a sixth if compared to the FM procedure. The improvement provided by the DMTS procedure is not so significant, but this is because most of the elements on the simulation reach their non-linear threshold, and, the more elements in the non-linear range, the more that the method requires the same computational cost as a FM procedure. This situation is reduced in large structures, where the elements that suffer damage are a minimal portion of the whole structure.

In Table [4] are also reported the corresponding time speedup for both cases without considering the time spent to generate the database since it is independent from the performed analysis.

Table 4: Computational cost for Full Multiscale Method, DMTS and DMCM

\begin{tabular}{ccccc}
\hline Type & Time $[s]$ & Memory $[k B]$ & NumberofActiveRVEs & TimeSpeedup \\
\hline Full Multiscale & 844.72 & 169788 & $484 / 484$ & - \\
DMTS & 646.39 & 92140 & $388 / 484$ & 1.307 \\
DMCM & 282.01 & 26696 & $0 / 484$ & 2.995 \\
\hline
\end{tabular}


We also provide in the following figures the evolution of $u_{y}, \sigma_{\min }, \varepsilon_{\max }, d_{e q}$ for each analyzed method at different level of deformation in $y$-direction. We can appreciate how the results obtained with DMTS and DMCM reflect the reference values calculated with FM.

Figure 18: Contour plot of $u_{y}$

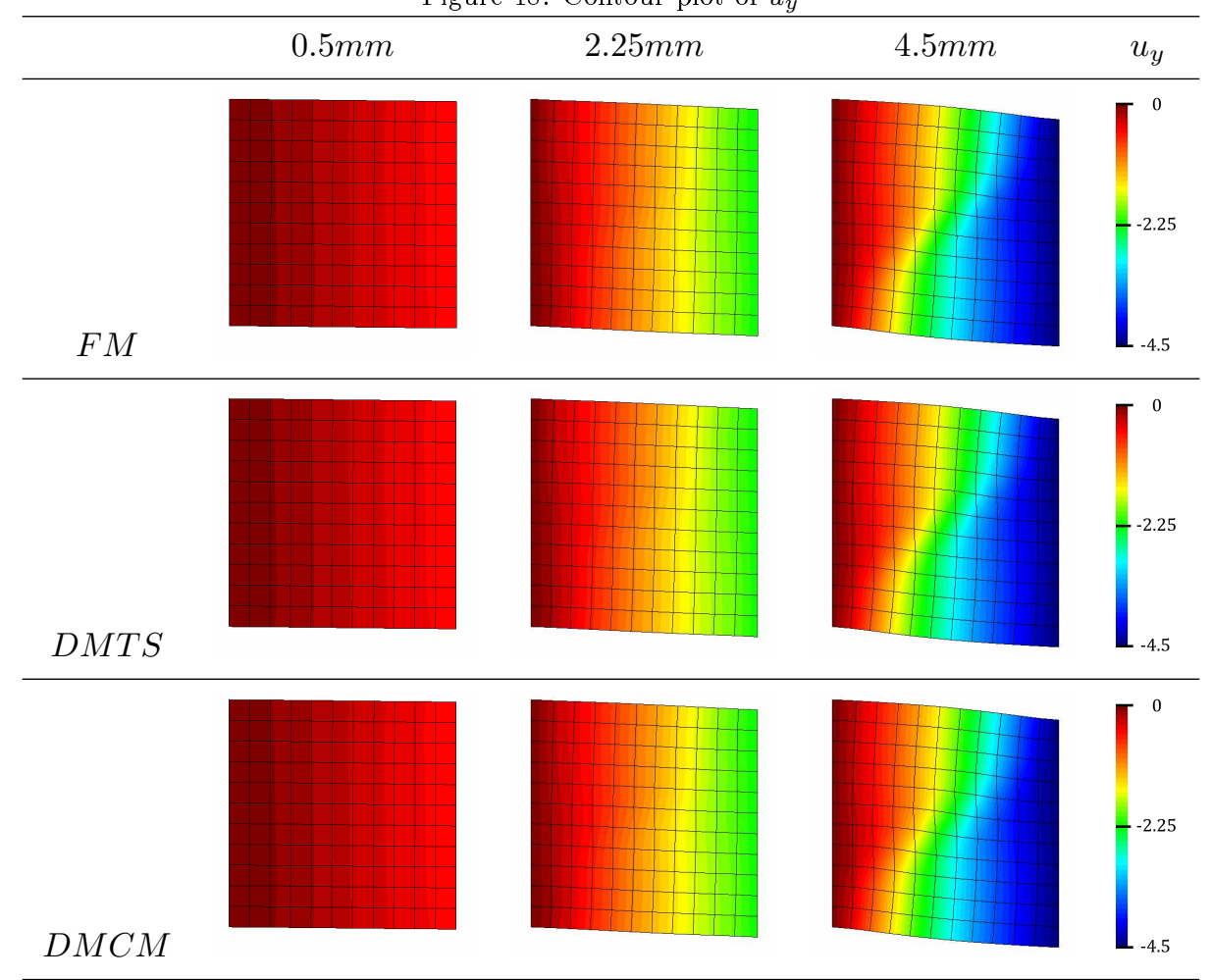


Figure 19: Contour plot of $\sigma_{\min }$

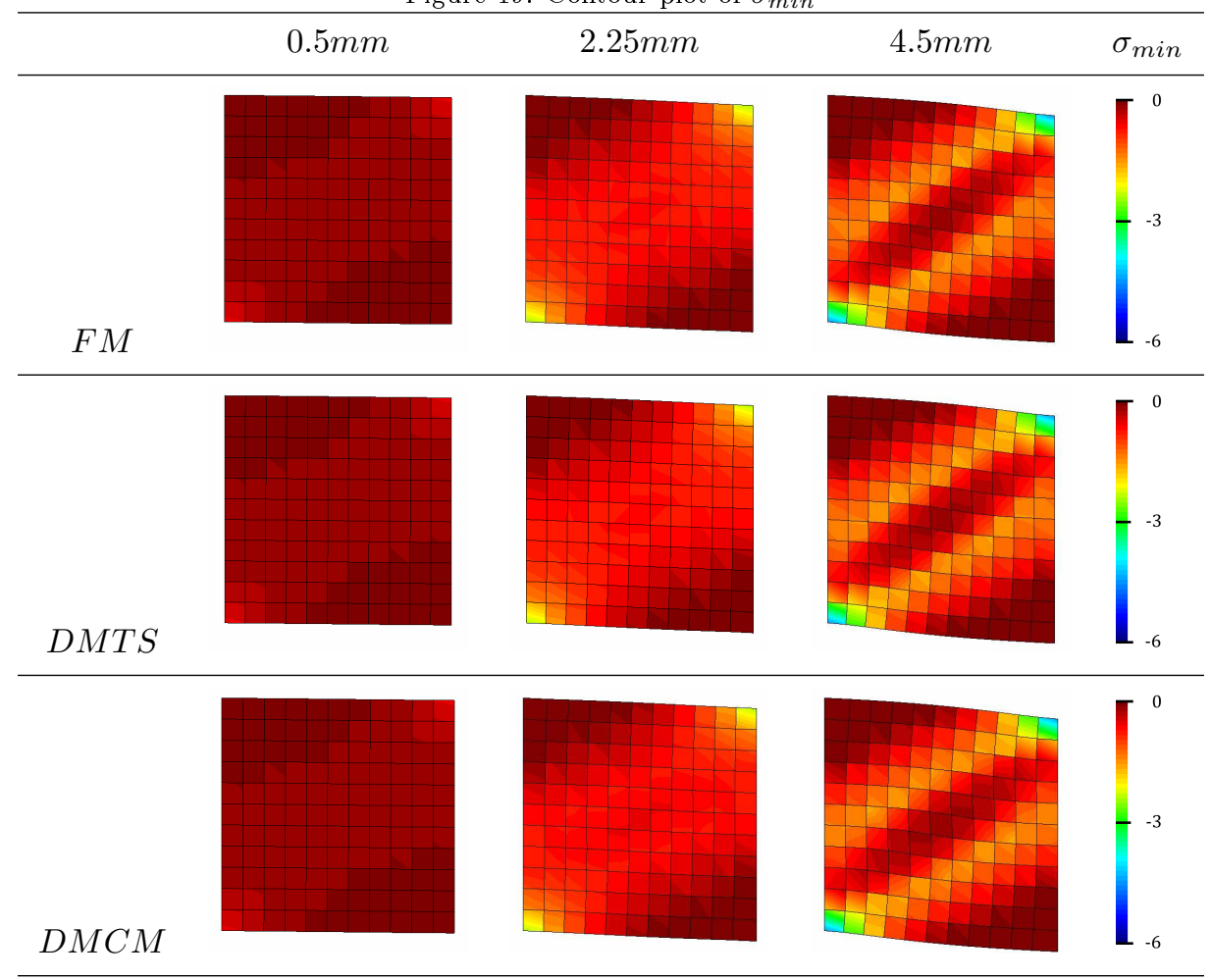


Figure 20: Contour plot of $\varepsilon_{\max }$

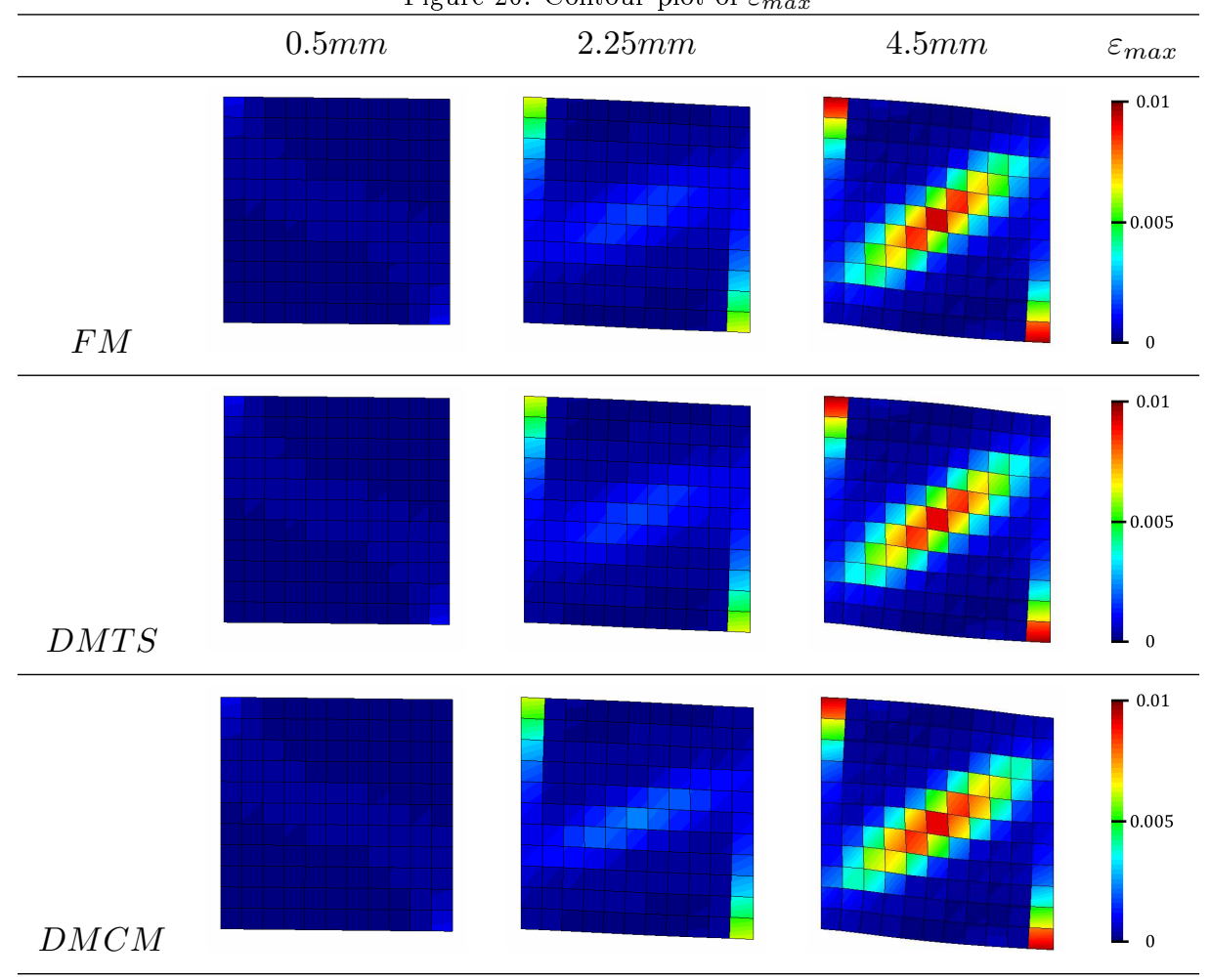


Figure 21: Contour plot of $d_{e q}$

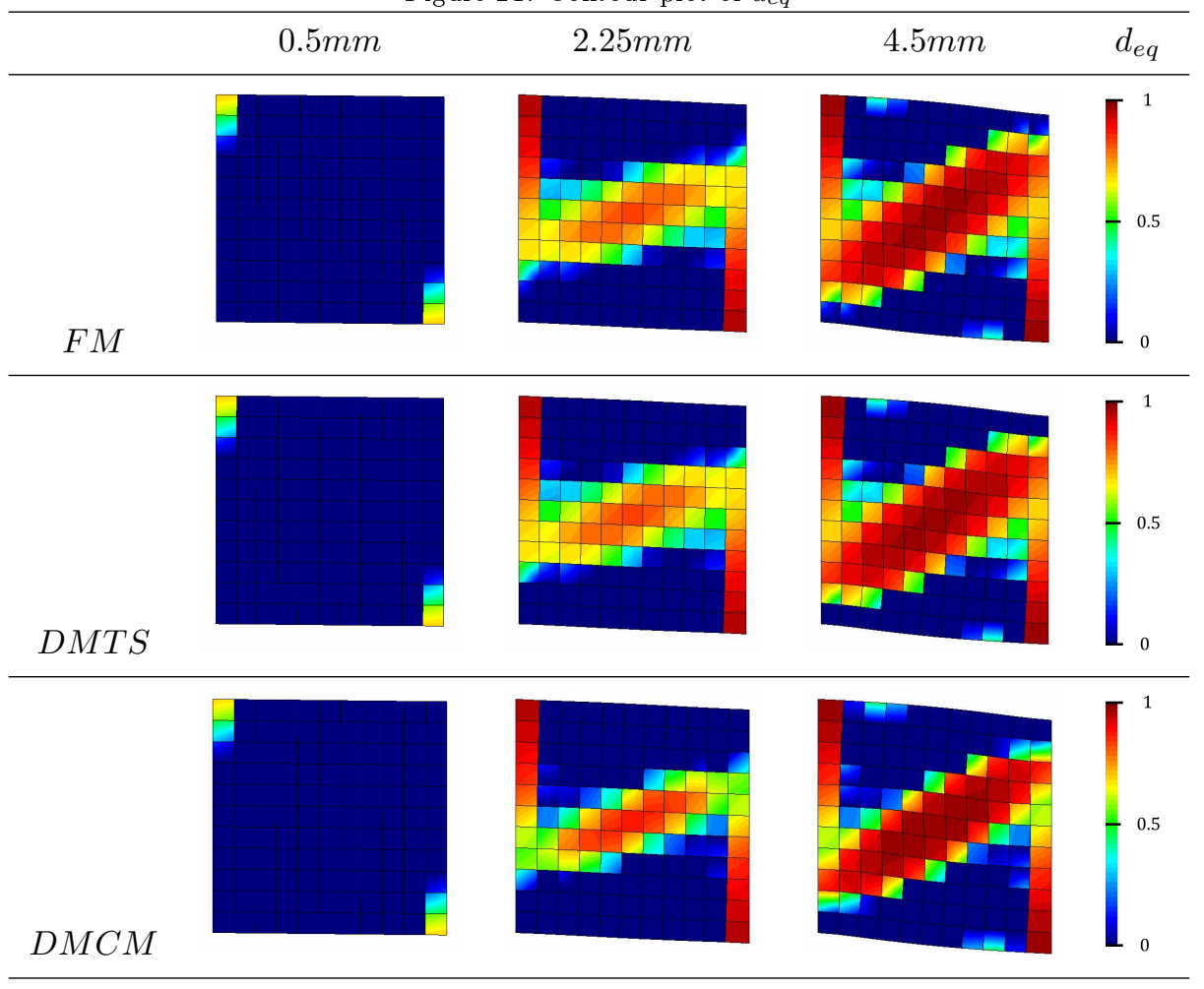

\subsection{Reinforced composite beam section}

In this section we analyze a reinforced composite beam section comparing the three proposed methods. The microscale used is a long fiber reinforced composite, contains F155 Epoxy Resin matrix and Carbon fiber (simulated as elastic material for simplicity). The geometry of the RVE was composed by 5 symmetric inclusions and the mesh used for the micro scale is composed by 272 small displacement linear elements with 305 nodes. The macroscale structure is composed by 1075 triangular elements and 678 nodes subjected to a vertical displacement $u_{y}$. Geometry and boundary conditions of the beam and RVE are described in the Figure [22]. The analysis was performed by using plane stress theory and considering $200 \mathrm{~mm}$ as thickness of the beam.

In this case, as in the section 4.1, the discretization parameter used to construct the stress-strain database is $m=8$ that required a minimum of 114 analyses of the RVE. While, the levels of damage stored for each direction are $[0.01,0.05,0.1,0.5,0.7,0.8$, $0.9,0.99,0.999]$. Each one of the analyses was carried out with a single standard PC equipped with an Intel Core i7-6700HQ CPU and 8 GB RAM. In Table [5] is reported the computational effort required for both DMTS and DMCM methods. 
Table 5: Computational cost for Database construction of composite RVE in case of DMTS and DMCM method

\begin{tabular}{ccc}
\hline Type & Time Full Database [s] & Memory Single RVE [MB] \\
\hline DMTS & 461 & 24.5 \\
DMCM & 1382 & 24.5
\end{tabular}

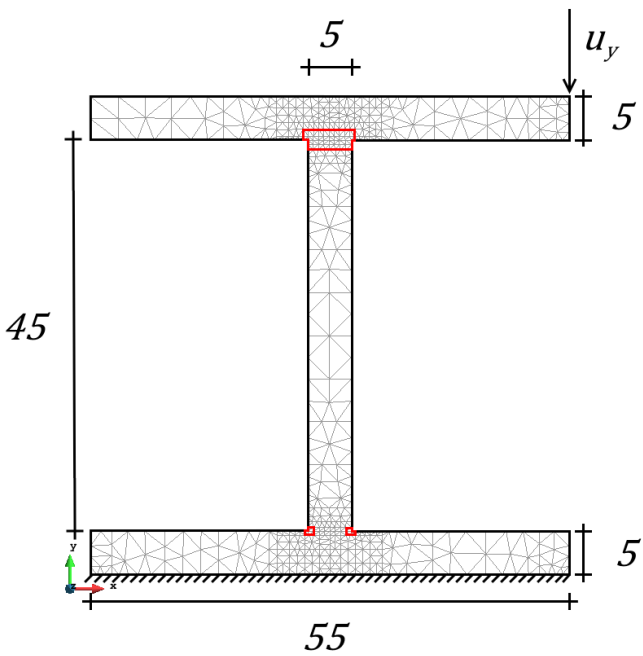

(a)

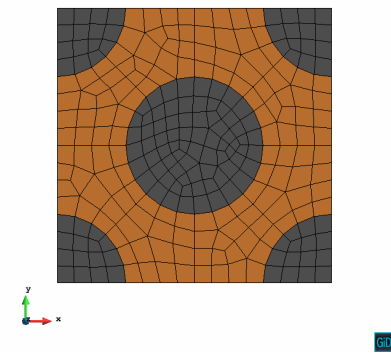

(b)

Figure 22: Geometry in [mm], boundary conditions and mesh discretization for beam and RVE models

Table 6: Material Properties of F115 Epoxy Resin

\begin{tabular}{cccccc}
\hline$E[M P a]$ & $\nu$ & $\sigma_{t}[M P a]$ & $G_{t}\left[\frac{J}{m^{2}}\right]$ & $\sigma_{c}[M P a]$ & $G_{c}\left[\frac{J}{m^{2}}\right]$ \\
\hline $3.24 \mathrm{e} 3$ & 0.32 & 80 & 0.73 & 240 & 2.19 \\
\hline
\end{tabular}

Table 7: Material Properties of Carbon fiber

\begin{tabular}{cc}
\hline$E[M P a]$ & $\nu$ \\
\hline $235 \mathrm{e} 3$ & 0.21 \\
\hline 2
\end{tabular}


In Figure [23] are reported the sections in the $\sigma_{x x}, \sigma_{y y}$ plane the elastic limit surface for $d e q=0.01$ and the stress evolution for $d_{e q}=0.1,0.5$ for the composite.

Each graph shows the stress tensor associated to a given damage level, as well as the damage parameter in the RVE at certain points of the graph. These show that failure begins and evolves in the matrix material in contact with fibers.

Another important result shown in Figure [23] is the lost of convexity of the stress curves, as well as the combination of hardening and softening behaviours, depending on the strains applied to it. This phenomena cannot be captured by a regular constitutive law, which makes imperative the use of multiscale procedures or discrete multiscale procedures such the ones proposed in this work. 


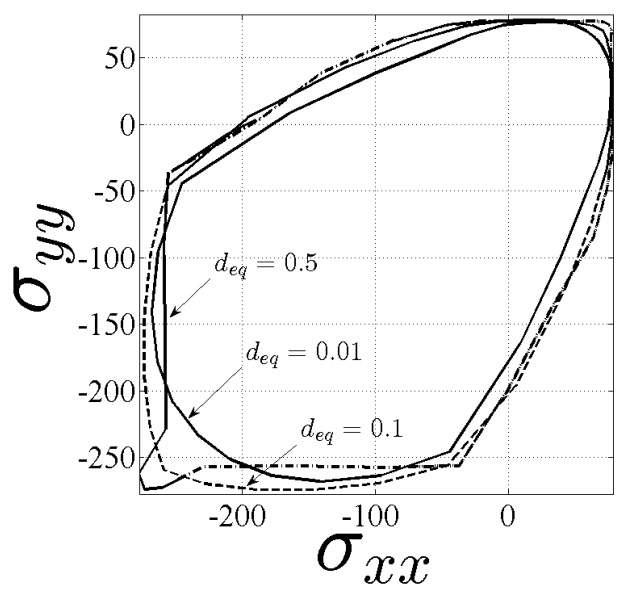

(a)

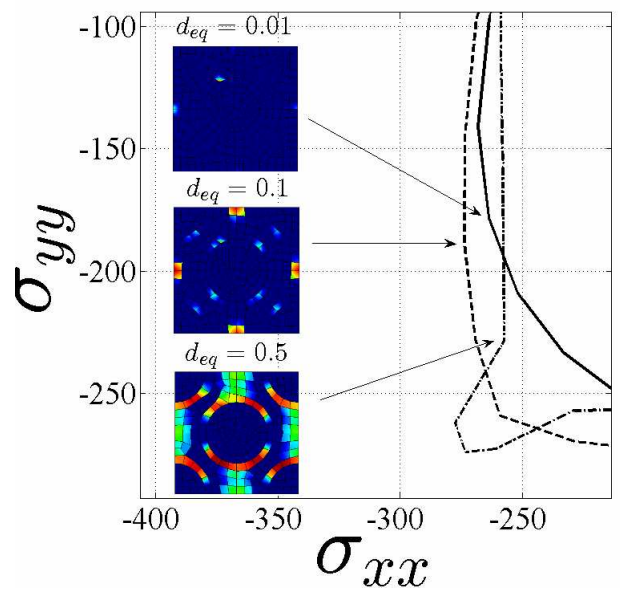

(c)

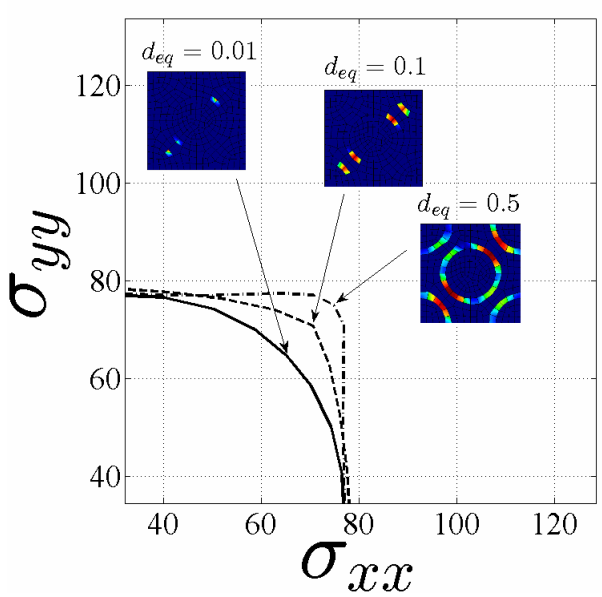

(b)

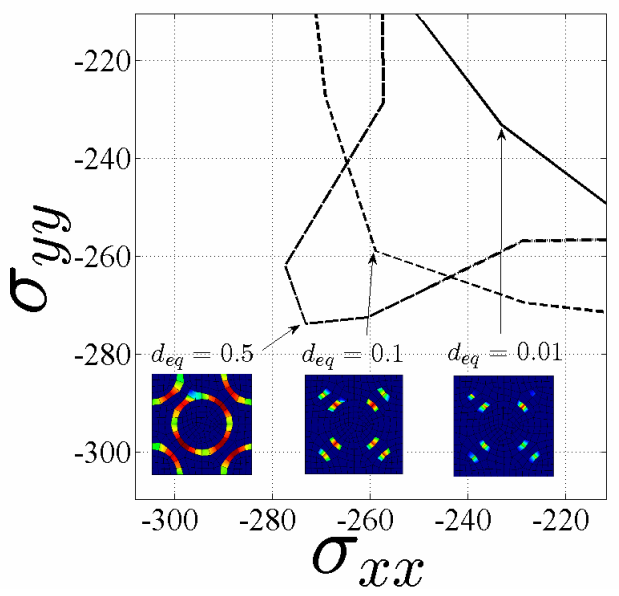

(d)

Figure 23: Composite Stress Evolution Surfaces

In order to reduce the computational cost of the numerical analysis, in case of Full Multiscale and DMTS methods, only 84 elements are considered with double scale, using elastic homogenized properties in the rest of the structure. As we can see in the Figure [24], Force-Displacement curves of DMTS and DMCM methods overlap and achieve the same maximum value of force of the FM2 case. Therefore, this result shows that the two procedures developed are capable of predicting the global structural response. However, our interest lays on obtaining the global response, as well as a good prediction of the failure region and failure mode of the structure. This is shown by the contour plots shown in Figure [27]. 


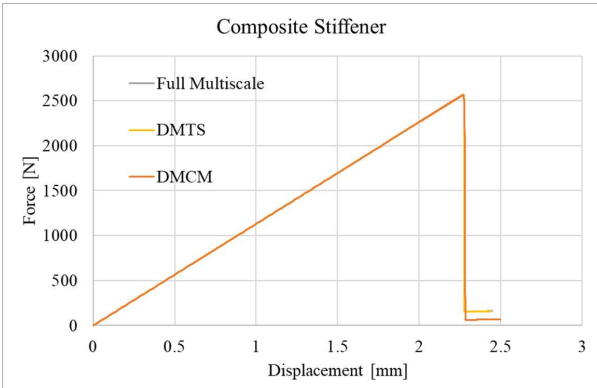

(a)

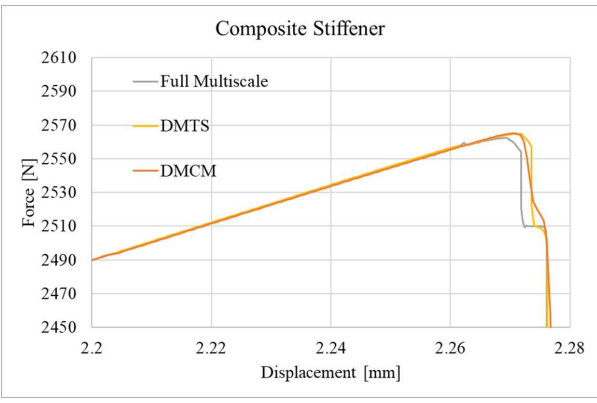

(b)

Figure 24: Force-Displacement evolution for the Ful Multiscale, DMTS and DMCM methods

Figure [25] shows the equivalent damage distribution on the beam and in the microstructure at the end of the analysis, corresponded to $u_{y}=2.5[\mathrm{~mm}]$.

In addition, regarding the DMTS method, in Figure [26] we proposed a portion of the beam where we can observe, in red, the total number of the elements that exceed the threshold surface. Indeed, during the analysis, the elements where the RVEs are generated increase proportionally to the non-linear behavior of the structure. 


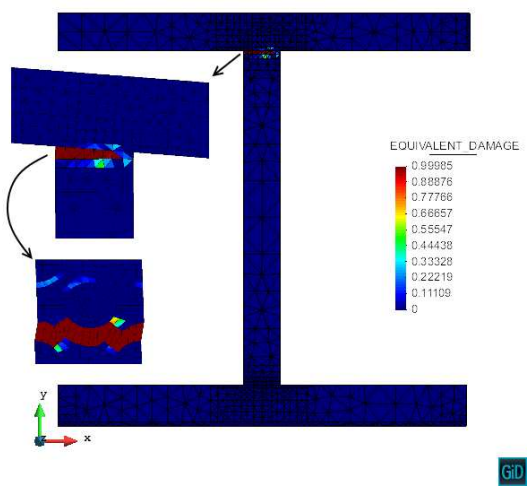

(a)

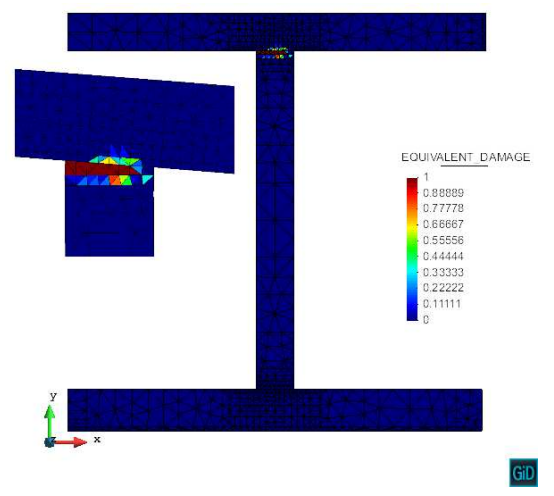

(b)

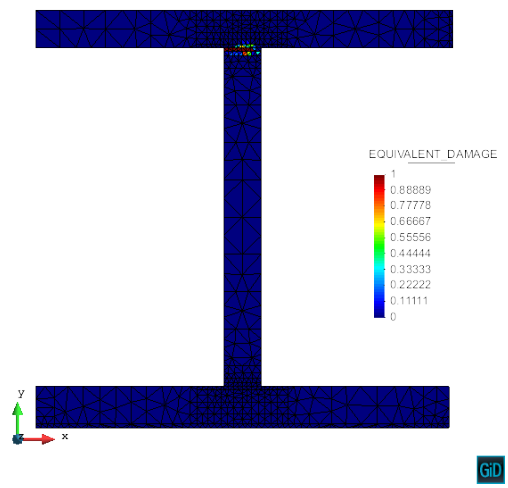

(c)

Figure 25: Equivalent damage distribution for Full Multiscale (a), DMTS (b), DMCM (c)

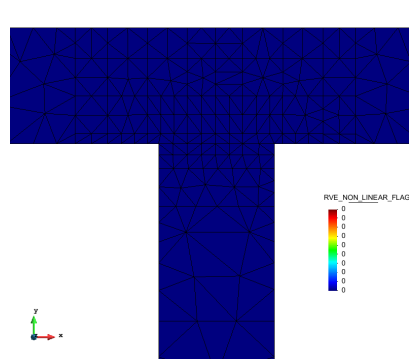

(a)

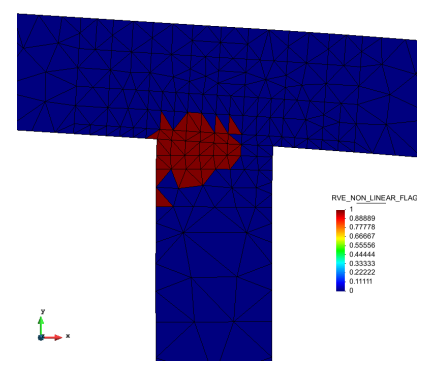

(b)

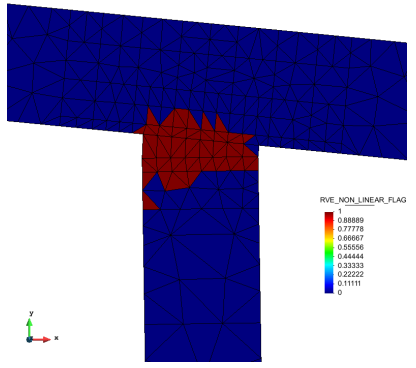

(c)

Figure 26: Macroscale elements that exceed the DMTS linear elastic boundary at $u_{y}=0.0[\mathrm{~mm}]$ (a), $u_{y}=2.27[\mathrm{~mm}](\mathrm{b})$ and $u_{y}=2.5[\mathrm{~mm}](\mathrm{c})$ 
Stress and strain distribution, when the $50 \%$ of maximum force $(1250[N])$ was achieved, are reported in the figures below in order to appreciate the correspondence between the reference FM results and the proposed methods.

Figure 27: Comparison between Full Multiscale, DMTS and DMCM for $d_{e q}, \sigma_{\max }, \sigma_{\min }$ and deformation $\varepsilon_{\max }, \underline{\varepsilon_{\text {min }}}$

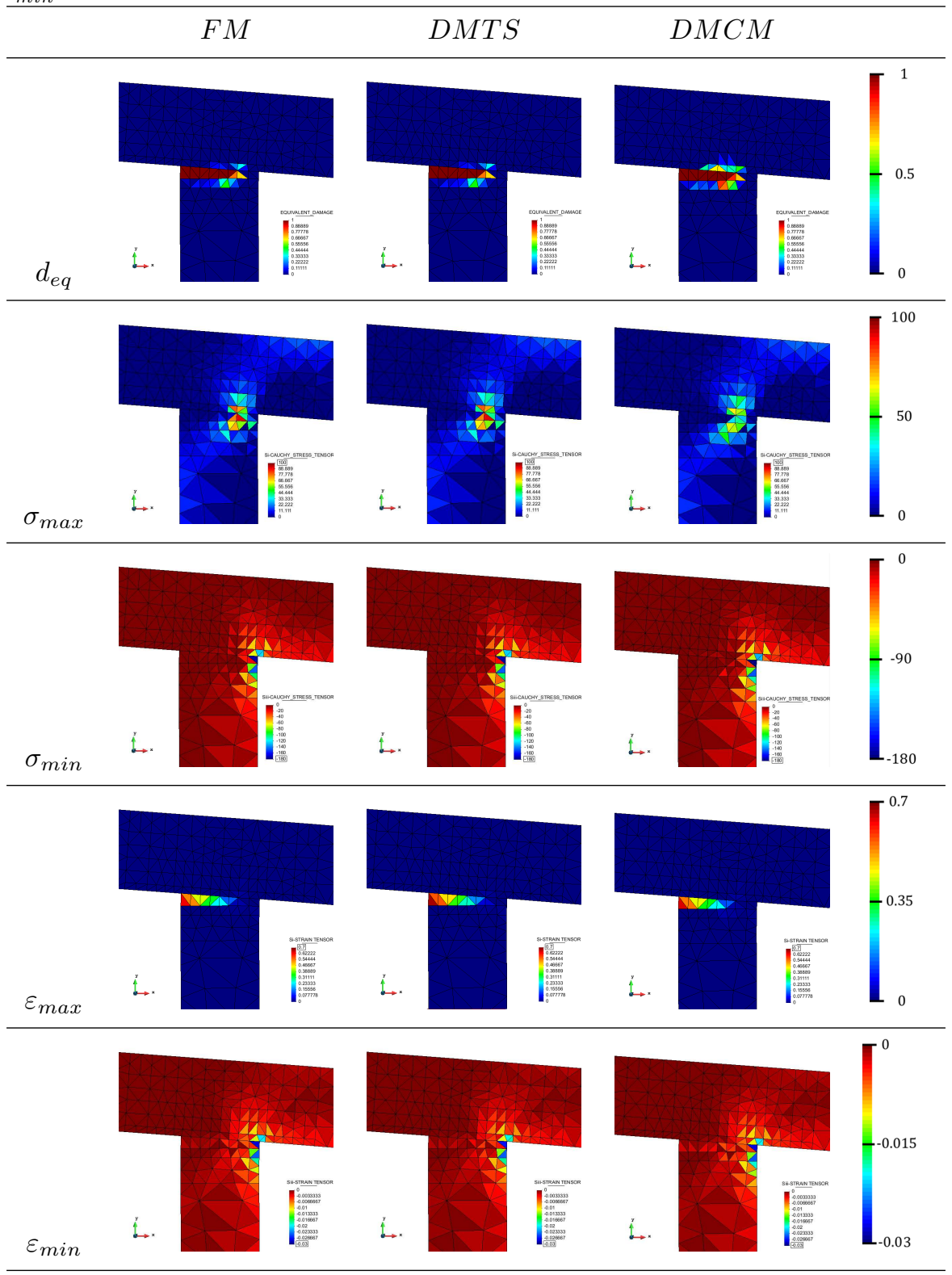


In case of reinforced composite beam, computational effort decreases significantly using the optimization method proposed in this paper. Indeed, as we can see in Table [8], we obtain a speedup of 4.7 and 661.2 for DMTS and DMCM method respectively. Each one of the analyses was carried out with a single standard PC equipped with an Intel Core i7-6700HQ CPU and 8 GB RAM.

The authors will remarks that, as in section 4.1, in Table [8] the reported time speedup not considers the time spent to generate the database described in Table [5].

Despite of the 70 RVEs generated in the DMTS, against the 84 of the FM, we are still having advantages using this method. Moreover, DMCM it is clearly faster than FM2 and DMTS as well. The linear interpolation of the RVE strain history can provide a nonlinear behavior of the macrostructure, able to reproduce crack initiation and propagation.

Table 8: Computational cost for Full Multiscale Method, DMTS and DMCM

\begin{tabular}{ccccc}
\hline Type & Time $[s]$ & Memory $[$ MB $]$ & Numberof ActiveRVEs & TimeSpeedup \\
\hline Full Multiscale & 40267.5 & 152.2 & $84 / 1075$ & - \\
DMTS & 8609.9 & 108.7 & $70 / 1075$ & 4.7 \\
DMCM & 60.9 & 33.1 & $0 / 1075$ & 661.2 \\
\hline
\end{tabular}

\section{Extension of the formulation to the $3 \mathrm{D}$ case}

The two procedures proposed in this work DMTS and DMCM have been implemented in a two-dimensional space. The extension of the formulation to the $3 \mathrm{D}$ case is quite straightforward, as the FE code implementation needs only some marginal changes in the parsing loop over the tags. Indeed, as in the 2D case, we can uniquely define the strain direction subdividing the interval $[0, \pi]$ of each dimension with the parameter $m \in[1, \infty)$. In that way we obtain $(2 \cdot m+1)^{5}$ sequence of 5 tags, one for each dimension, covering all the 5D-Hypersphere. Also in this case, we can reduce the total amount of strain histories, considering the superposition of the vectors, to $(((2 \cdot m) \cdot(m-1)+2) \cdot(m-$ $1)+2) \cdot(m-1)+2) \cdot(m-1)+2$.

Obviously, compared to the 2D the computational costs increase significantly in terms of number of analysis needed to cover all the strain spaces, as we can see in the Figure [28]. Despite the increase on the computational cost required to generate a material 3D database, we can obtain enormous advantages during the a-posteriori analysis at the structural level. Time and memory consumption of the macroscale analysis, thanks to the DMCM technique, become equal to a linear elastic constitutive law because the procedure only requires the interpolation of the stress-strain database values. 


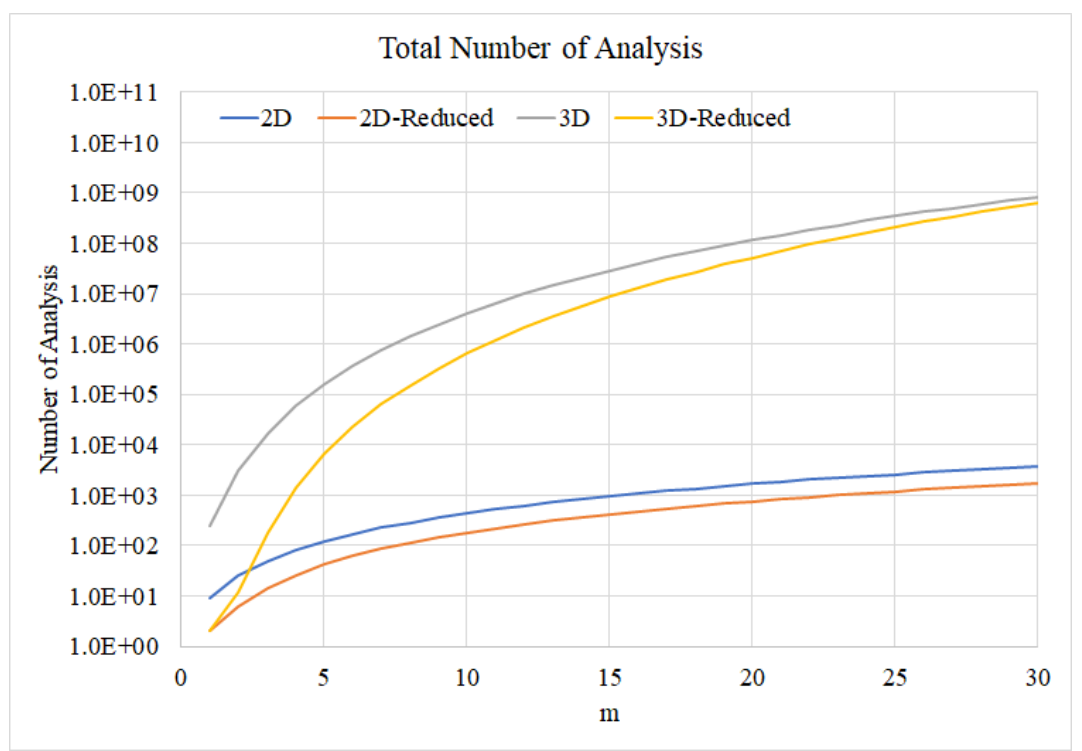

Figure 28: Comparison of Number of Analysis VS discretization parameter for 3D and 2D Case in both classical and reduced form

\section{Conclusions}

First order multiscale homogenization method can fully describe both linear and nonlinear behavior of complex microstructures, and the impact they have on the macroscale response. However, the computational cost required to analyze large structures is not negligible. In the last years, many methods have been developed to overcome this problem. In this paper, the authors propose two techniques that can provide a significant speed-up compared to the classical FM without loss of accuracy on the final results. By conducting a previous finite element analysis of the RVE, the DMTS procedure gives the linear elastic threshold. Once this is reached, it becomes necessary to solve the RVE in that given material point to obtain the non-linear material performance.

On the other hand, the Discrete Multiscale Constitutive Model (DMCM) comes as a natural extension of the DMTS. With this procedure, during the initial analysis of the RVE it is computed, not only the threshold surface in which the non-linear behavior starts, but the surfaces associated to different damage values. Aftwerards, the non-linear multiscale anaysis can be done using only the values stored in the database, without having to solve the RVE at any time.

The keys of both methods are the precomputed Stress-Strain Data Base and the equivalent damage parameter, essential to reconstruct the correct behavior of the structure. Moreover, the JSON format used to construct the database makes all the data more readable, faster and easier to manage. A fracture-energy-based regularization procedure is also proposed in order to remove the dependence of the results on macro and micro-scale mesh size. Thanks to the proposed regularization, the Stress-Strain Data Base becomes 
independent from the macro-scale mesh discretization. DMTS and DMCM methods are validated by a simple benchmark analysis and by a more complex analysis of a reinforced composite beam, showing the capability and robustness of these techniques. The achieved speed-up of more than 600 times respect to FM and the accuracy of the results, justifies the time spent to obtain the Stress-Strain Data Base.

\section{Acknowledgments}

This work has been supported by European Research Council through of Advanced Grant: ERC-2012-AdG320815 (COMP-DES-MAT), by the Spanish Ministerio de Economia y Competividad through the project: MAT2014-60647-R (OMMC) and BIAS201567807-R (RESCICLO), by European Union EU-H2020 (Agreement No 690638) and the People's Republic of China (Agreement No [2016]92) (ECOCOMPASS). All this support is gratefully acknowledged. 


\section{References}

[1] E. Car, S. Oller, E. Oñate, An anisotropic elastoplastic constitutive model for large strain analysis of fiber reinforced composite materials, Computer Methods in Applied Mechanics and Engineering 185 (2) (2000) 245 - 277, ISSN 0045-7825, doi https://dx.doi.org/10.1016/S0045- 7825(99)00262-5, URL http://www.sciencedirect.com/science/article/pii/S0045782599002625.

[2] F. Rastellini, S. Oller, O. Salomón, E. Oñate, Composite materials non-linear modelling for long fibre-reinforced laminates: Continuum basis, computational aspects and validations, Computers \& Structures 86 (9) (2008) 879 - 896, ISSN 0045-7949, doi https://dx.doi.org/ 10.1016/j.compstruc.2007.04.009, URL http://www.sciencedirect.com/science/article/pii/ S0045794907001642, composites.

[3] X. Martinez, S. Oller, F. Rastellini, A. H. Barbat, A numerical procedure simulating RC structures reinforced with FRP using the serial/parallel mixing theory, Computers \& Structures 86 (15) (2008) 1604 - 1618, ISSN 0045-7949, doi https://dx.doi.org/10.1016/j.compstruc.2008.01.007, URL http: //www.sciencedirect.com/science/article/pii/S0045794908000096.

[4] E. Sanchez-Palencia, Non-Homogeneous Media and Vibration Theory, 127, Springer-Verlag Berlin Heidelberg, 1 edn., ISBN 978-3-540-39317-7, doi https://dx.doi.org/10.1007/3-540-10000-8, 1980.

[5] P. M. Suquet, Local and Global Aspects in the Mathematical Theory of Plasticity, Plasticity Today (1985) 279-309URL https://ci.nii.ac.jp/naid/10009833388/en/.

[6] J. Hernández, J. Oliver, A. Huespe, M. Caicedo-Silva, J. Cante, High-performance model reduction techniques in computational multiscale homogenization, Computer Methods in Applied Mechanics and Engineering 276 (2014) 149-189.

[7] F. Otero, X. Martinez, S. Oller, O. Salomon, Study and prediction of the mechanical performance of a nanotube-reinforced composite, Composite Structures 94 (2012) 2920-2930.

[8] F. Otero, S. Oller, X. Martinez, O. Salomon, Numerical homogenization for composite materials analysis. Comparison with other micro mechanical formulations, Composite Structures 122 (2015) $405-416$.

[9] L. G. N. R. D. Quinteros, S. Oller, Nonlinear homogenization techniques to solve masonry structures problems., Composite Structures 94 (2) (2012) 724-730.

[10] S. Oller, J. Miquel Canet, F. Zalamea, Composite Material Behavior Using a Homogenization Double Scale Method, Journal of Engineering Mechanics-asce - J ENG MECH-ASCE 131, doi https://dx.doi.org/10.1061/(ASCE)0733-9399(2005)131:1(65).

[11] E. Car, F. Zalamea, S. Oller, J. Miquel, E. Oñate, Numerical simulation of fiber reinforced composite materials - Two procedures, International journal of solids and structures 39 (2002) 1967-1986.

[12] J. Yvonnet, Q.-C. He, The reduced model multiscale method (R3M) for the non-linear homogenization of hyperelastic media at finite strains, Journal of Computational Physics 223 (2007) 341-368.

[13] I. Temizer, P. Wriggers, An adaptive method for homogenization in orthotropic nonlinear elasticity, Computer Methods in Applied Mechanics and Engineering 196 (2007) 3409-3423.

[14] F. Otero, X. Martinez, S. Oller, O. Salomón, An efficient multi-scale method for non-linear analysis of composite structures, Composite Structures 131 (2015) 707 - 719, ISSN 0263-8223, doi https://doi.org/10.1016/j.compstruct.2015.06.006, URL http://www.sciencedirect.com/science/ article/pii/S0263822315004699.

[15] M. Geers, V. Kouznetsova, W. Brekelmans, Multi-scale first-order and second-order computational homogenization of microstructures towards continua, International Journal for Multiscale Computational Engineering 1 (4) (2003) 371-386, ISSN 1543-1649, doi 10.1615/IntJMultCompEng.v1.i4.

[16] T. Kanit, S. Forest, I. Galliet, V. Mounoury, D. Jeulin, Determination of the size of the representative volume element for random composites: statistical and numerical approach, International Journal of Solids and Structures 40 (13) (2003) 3647 - 3679, ISSN 0020-7683, doi https://dx. doi.org/10.1016/S0020-7683(03)00143-4, URL http://www.sciencedirect.com/science/article/ $\mathrm{pii/S0020768303001434.}$

[17] M. Jiang, M. Ostoja-Starzewski, I. Jasiuk, Scale-dependent bounds on effective elastoplastic response of random composites, Journal of the Mechanics and Physics of Solids 49 (2001) 655-673.

[18] M. Jiang, I. Jasiuk, M. Ostoja-Starzewski, Apparent elastic and elastoplastic behavior of periodic composites, International Journal of Solids and Structures 39 (2002) 199-212.

[19] M. Ostoja-Starzewski, Ostoja-Starzewski, M.: Material spatial randomness: from statistical to representative volume element., Probabilistic Engineering Mechanics 21 (2006) 112-132.

[20] R. Hill, A self-consistent mechanics of composite materials, Journal of the Mechanics and Physics of Solids 13 (4) (1965) 213 - 222, ISSN 0022-5096, doi https://dx.doi.org/10.1016/0022-5096(65) 90010-4, URL http://www.sciencedirect.com/science/article/pii/0022509665900104. 
[21] E. de Souza Neto, R. Feijóo, EA de Souza Neto, RA Feijoo. Variational Foundations of Multi-Scale Constitutive Models of Solid: Small and Large Strain Kinematical Formulation, LNCC R\&D Report

[22] J. O. J. M. Ortolano J., A. Hernández, A Comparative Study on Homogenization Strategies for Multi-Scale Analysis of Materials, Monograph CIMNE 135.

[23] S. Ghosh, K. Lee, S. Moorthy, Two scale analysis of heterogeneous elastic-plastic materials with asymptotic homogenization and Voronoi cell finite element model, Computer Methods in Applied Mechanics and Engineering 132 (1) (1996) 63 - 116, ISSN 0045-7825, doi https://dx.doi. org/10.1016/0045-7825(95)00974-4, URL http://www.sciencedirect.com/science/article/pii/ 0045782595009744.

[24] JSON (JavaScript Object Notation), URL http://www.json.org/, 1999.

[25] M. Petracca, L. Pelà, R. Rossi, S. Zaghi, G. Camata, E. Spacone, Micro-scale continuous and discrete numerical models for nonlinear analysis of masonry shear walls, Construction and Building Materials 149 (2017) 296 - 314, ISSN 0950-0618, URL https://dx.doi.org/10.1016/j.conbuildmat.2017. 05.130.

[26] M. Cervera, M. Chiumenti, Mesh objective tensile cracking via a local continuum damage model and a crack tracking technique, Computer Methods in Applied Mechanics and Engineering 196 (1) (2006) 304-320.

[27] Z. Bažant, B. Oh, Crack Band Theory for Fracture of Concrete, Bordas-Dunod, URL http:// books.google.it/books?id=x9citwAACAAJ, 1983.

[28] J. Oliver, A consistent characteristic length for smeared cracking models, International Journal for Numerical Methods in Engineering 28 (2) (1989) 461-474.

[29] S. Oller, Fractura Mecánica: Un Enfoque Global, Ediciones CIMNE y UPC, 2001.

[30] M. Petracca, P. Luca, R. Riccardo, O. Sergio, C. Guido, S. Enrico, Regularization of first order computational homogenization for multiscale analysis of masonry structures, Computational Mechanics 57 (2) (2016) 257-276, ISSN 1432-0924, URL http://dx.doi.org/10.1007/s00466-015-1230-6.

[31] P. R. L. Pelà, M. Cervera, Continuum damage model for orthotropic materials: Application to masonry., Computer Methods in Applied Mechanics and Engineering 200 (9) (2011) 917-930.

[32] P. R. L. Pelà, M. Cervera, An orthotropic damage model for the analysis of masonry structures, Construction and Building Materials 41 (2013) 957-967.

[33] J. G. R. P. B. Lourenço, Multisurface interface model for analysis of masonry structures, Journal of engineering mechanics 123 (7) (1997) 660-668.

[34] W. A. M. B. V. Kouznetsova, M. G. D. Geers, Multi-scale constitutive modelling of heterogeneous materials with a gradient-enhanced computational homogenization scheme, International Journal for Numerical Methods in Engineering 54 (8) (2002) 1235-1260.

[35] W. A. M. B. V. Kouznetsova, M. G. D. Geers, Multi-scale second-order computational homogenization of multi-phase materials: a nested finite element solution strategy, Computer Methods in Applied Mechanics and Engineering 193 (2004) 5525-5550.

[36] E. O. P. Dadvand, R. Rossi, An object-oriented environment for developing finite element codes for multi-disciplinary applications, Archives of computational methods in engineering 17 (3) (2010) $253-297$.

[37] GiD: The personal pre and post preprocessor, URL http://www.gid.cimne.upc.es/, 2002.

[38] M. Cervera, L. Pelà, R. Clemente, P. Roca, A crack-tracking technique for localized damage in quasi-brittle materials, Engineering Fracture Mechanics 77 (13) (2010) 2431-2450.

[39] M. Cervera, J. Oliver, R. Faria, Seismic evaluation of concrete dams via continuum damage models, Earthquake engineering \& structural dynamics 24 (9) (1995) 1225-1245.

[40] R. Faria, J. Oliver, M. Cervera, A strain-based plastic viscous-damage model for massive concrete structures, International Journal of Solids and Structures 35 (14) (1998) 1533-1558.

[41] J. Y. Wu, J. Li, R. Faria, An energy release rate-based plastic-damage model for concrete, International Journal of Solids and Structures 43 (3) (2006) 583-612.

[42] J. Lubliner, J. Oliver, S. Oller, E. Oñate, A plastic-damage model for concrete, International Journal of solids and structures 25 (3) (1989) 299-326.

[43] D. V. Oliveira, P. B. Lourenço, Implementation and validation of a constitutive model for the cyclic behaviour of interface elements, Computers \& structures 82 (17) (2004) 1451-1461.

[44] J. C. Simo, M. Rifai, A class of mixed assumed strain methods and the method of incompatible modes, International Journal for Numerical Methods in Engineering 29 (8) (1990) 1595-1638.

[45] P. Dadvand, R. Rossi, M. Gil, X. Martorell, J. Cotela, E. Juanpere, S. R. Idelsohn, E. Oñate Migration of a generic multi-physics framework to HPC environments, Computers \& Fluids 80 (2013) 301-309. 
[46] P. Dadvand, R. Rossi, E. Oñate, An object-oriented environment for developing finite element codes for multi-disciplinary applications, Archives of computational methods in engineering 17 (3) (2010) $253-297$.

[47] P. Roca, M. Cervera, G. Gariup, L. Pelà, Structural Analysis of Masonry Historical Constructions Classical and Advanced Approaches, Archives of Computational Methods in Engineering 17 (3) (2010) 299-325, ISSN 1134-3060.

[48] L. Pelà, M. Cervera, S. Oller, M. Chiumenti, A localized mapped damage model for orthotropic materials, Engineering Fracture Mechanics 124-125 (2014) 196-216, ISSN 0013-7944, URL http: //www.sciencedirect.com/science/article/pii/S0013794414001386.

[49] L. Pelà, M. Cervera, P. Roca, An orthotropic damage model for the analysis of masonry structures, Construction and Building Materials 41 (2013) 957-967, ISSN 0950-0618, URL http: //www.sciencedirect.com/science/article/pii/S0950061812004837.

[50] S. Saloustros, L. Pelà, M. Cervera, A crack-tracking technique for localized cohesive-frictional damage, Engineering Fracture Mechanics 150 (2015) 96-114, ISSN 0013-7944, URL http://dx.doi. org/10.1016/j.engfracmech.2015.10.039.

[51] A. Anthoine, Homogenization of periodic masonry: plane stress, generalized plane strain or 3D modelling?, International Journal for Numerical Methods in Biomedical Engineering 13 (5) (1997) $319-326$.

[52] D. Addessi, E. Sacco, Nonlinear analysis of masonry panels using a kinematic enriched plane state formulation, International Journal of Solids and Structures 90 (2016) 194-214.

[53] M. Petracca, L. Pelà, R. Rossi, S. Oller, G. Camata, E. Spacone, Multiscale computational first order homogenization of thick shells for the analysis of out-of-plane loaded masonry walls, Computer Methods in Applied Mechanics and Engineering 315 (2017) 273-301.

[54] S. Saloustros, L. Pelà, M. Cervera, P. Roca, Finite element modelling of internal and multiple localized cracks, Computational Mechanics (2016) 1-18. 